\title{
Modelling fibre laydown and web uniformity in nonwoven fabric
}

\author{
F Battocchio and M P F Sutcliffe \\ Department of Engineering, University of Cambridge, Trumpington Street, \\ Cambridge, CB2 1PZ, UK \\ E-mail: francesco.battocchio@gmail.com
}

August 2016

\begin{abstract}
The mechanical and functional performance of nonwoven fabric critically depends on the fibre architecture. The fibre laydown process plays a key role in controlling this architecture. The fibre dynamic behaviour during laydown is studied through a finite element model which describes the role of the parameters in defining the area covered by a single fibre when deposited on the conveyor belt. The path taken by a fibre is described in terms of the radius of gyration, which characterises the area covered by the fibre in the textile, and the spectrum of curvature, which describes the degree of fibre looping as a function of the arc length. Starting from deterministic and idealised fibre curvature spectra, stochastic Monte Carlo simulations are undertaken to generate full nonwoven web samples and reproduce the uniformity of fibre density. A novel image analysis technique that allows measurement of the uniformity of real spunbonded nonwoven samples from images of textiles is used to confirm the validity of the model. It is shown that the main parameter that governs the fibre density uniformity is the ratio of the fibre spinning velocity to the velocity of conveyor belt, while fibre oscillations prior to deposition play a secondary role.
\end{abstract}

Keywords: Nonwovens, spunbonding, fibre dynamics, uniformity, image processing Submitted to: Modelling Simul. Mater. Sci. Eng.

\section{Introduction}

Understanding the fibre architecture generated by the laydown of continuous slender fibres on a moving collecting surface is of great interest in the nonwoven fabric industry, and in particular in the production of spunbonded nonwoven fabrics. The spunbonding process $[1,2]$ is a widespread method for the production of low-density nonwovens in which thousands of fibres are firstly spun by an accelerated air flow and progressively solidified, then randomly oscillated by a turbulent air flow in a diffuser, and finally deposited as a web on to a porous moving belt, as shown in Figure 1a. The geometry 
assumed by the fibres during the laydown process influences critical aspects of the material such as, at the micro-scale, the fibre-bond properties and, at the macro-scale, the homogeneity of the web and the material anisotropy. For instance, the motion of the belt establishes a preferential fibre direction along the machine direction (MD) [3, 4], and a lower fibre orientation distribution along the cross direction (CD), which results in an anisotropy of the mechanical properties [5]. At present, despite the crucial role of the fibre geometry in the nonwoven web, the number of studies of fibre laydown in the literature is very limited.

In their series of papers, Hearle and co-workers carried out pioneering work to investigate the form assumed by threads laid onto a moving belt $[6,7,8]$. They showed that, for a stationary conveyor belt, the thread forms concentric circular coils that superimpose over each other, with a diameter that depends on the feeding height and on the material properties such as density and bending stiffness. In particular, it was found that the feeding height influences the buckling regime: from compression-induced buckling for low heights, to self-weight induced buckling for large heights. For the case of a moving conveyor belt the geometry of the thread can be represented by a modified cycloid for feed-to-belt speed ratios greater than 2 , while for lower values, i.e. with increasing belt velocity, the looping progressively disappears and the thread assumes a wavy profile. Simplified geometric models have been introduced to simulate the fibre trajectory on the conveyor belt in the spunbonding process $[19,20,21]$. They were developed as an alternative to a more detailed model of fibre behaviour in spunbonding process $[22,23]$, due to the excessively high computational time required by the simulations. In this class of models the physics of fibre laydown is neglected, and the fibre profile is described as a parametric curve in which looping around a reference point is imposed at the level of curvature, and the effect of turbulence is modelled as a random Wenier process $[19,24,21]$. The main limitation of non-physically based stochastic models is that model parameters cannot be measured and are difficult to calibrate, which limit applications to the real industrial process.

The physics of laydown of a thread has been studied also through numerical models, although most of these works refer to a viscous thread deposited either on a stationary $[9,10]$, or a moving surface $[11,12]$. Since the periodic coiling pattern that a viscous thread generates on a moving substrate resembles the stitches of a sewing machine, the system has come to be known as the fluid-mechanical sewing machine (FMSM). Later on the analysis has been extended to an elastic thread, and the term elastic sewing machine (ESM) was adopted, as the pattern of the thread in the two systems was strikingly similar [13].

The first numerical study of an elastic rope which is deployed on a stationary surface is from Mahadevan [14], in terms of the Kirchhoff-Love equations for the motion of an elastic rod capable of large bending and twisting deformations [15]. It was shown that when the coiling is dominated either by inertia or gravity, the stiffness of the rope is relatively unimportant for the geometry assumed during the laydown. The radius of coiling decreases with the weight of the rope, and increases with the feed velocity in the 
inertial regime. Although this was the first numerical solution of a flexible rod on a static substrate, it was biased by a sign error in the inertial terms which was eliminated in [16]. On the other hand, for a moving substrate, the ESM referred typically to a system in which an elastic thread of polymeric material of diameter of the order of $1 \mathrm{~mm}$ is deployed with feed velocity $1-30 \mathrm{~cm} / \mathrm{s}$ onto a surface which is moving at comparable velocity $[13,17]$. The behaviour of the thread is governed by the relative contribution of the elastic forces which generated in the deformed thread, and is divided into three regimes: (i) elastic, when gravity and inertia are negligible relative to the elastic forces, (ii) gravity, in which the elastic forces are balanced by gravity, and (ii) inertial, when elastic forces are balanced by inertia. In the gravity regime, which takes place at lower belt speeds, the thread produces regular coils. If the belt speed is increased above a critical value, an inertial regime is established with a semi-random pattern being produced. [13].

In nonwoven production, fibre laydown has a higher degree of complexity since (i) the fibre diameter is $5-20 \mu \mathrm{m}$, (ii) the fibre is oscillating before deposition as a result of turbulence, and (iii) a vertical drag force exists that drives the fibre towards the belt. Under this conditions, the authors of this paper have recently described how a fibre with diameter $50 \mu \mathrm{m}$ in the diffuser behaves effectively as a string in which the work done by the air drag force accounts for more than 90 percent of the total work done on the fibre, while the work done by the bending forces is three orders of magnitude lower and therefore can be neglected [25, 18].

In the present work we extend the study to the fundamentals of fibre laydown on to a moving belt, and we simulate full scale nonwoven webs. This paper is divided into two parts. In the first part, the dynamics of the laydown of a single fibre onto a moving plane is solved in detail through a physically-based finite element model. In the second part of the paper, starting from a deterministic spectrum of curvature of a simulated fibre profile, artificial fibre webs are generated through stochastic Monte Carlo simulations. Randomness in the fibre behaviour is introduced in the spectrum of curvature, to simulate the effect of turbulence. The modelled uniformity of artificial webs is then compared to that of real spunbonded webs through a new image analysis technique. 


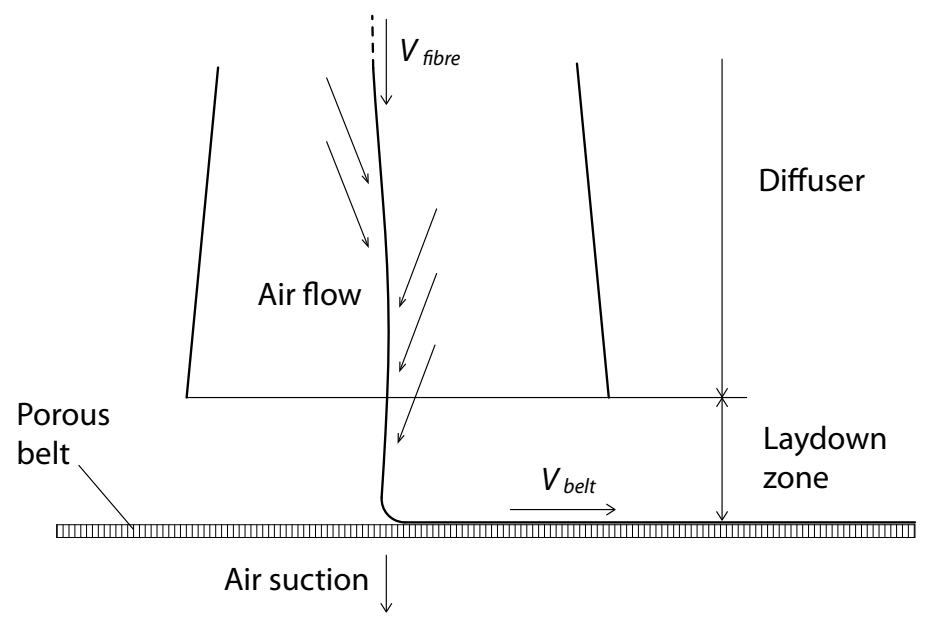

(a)

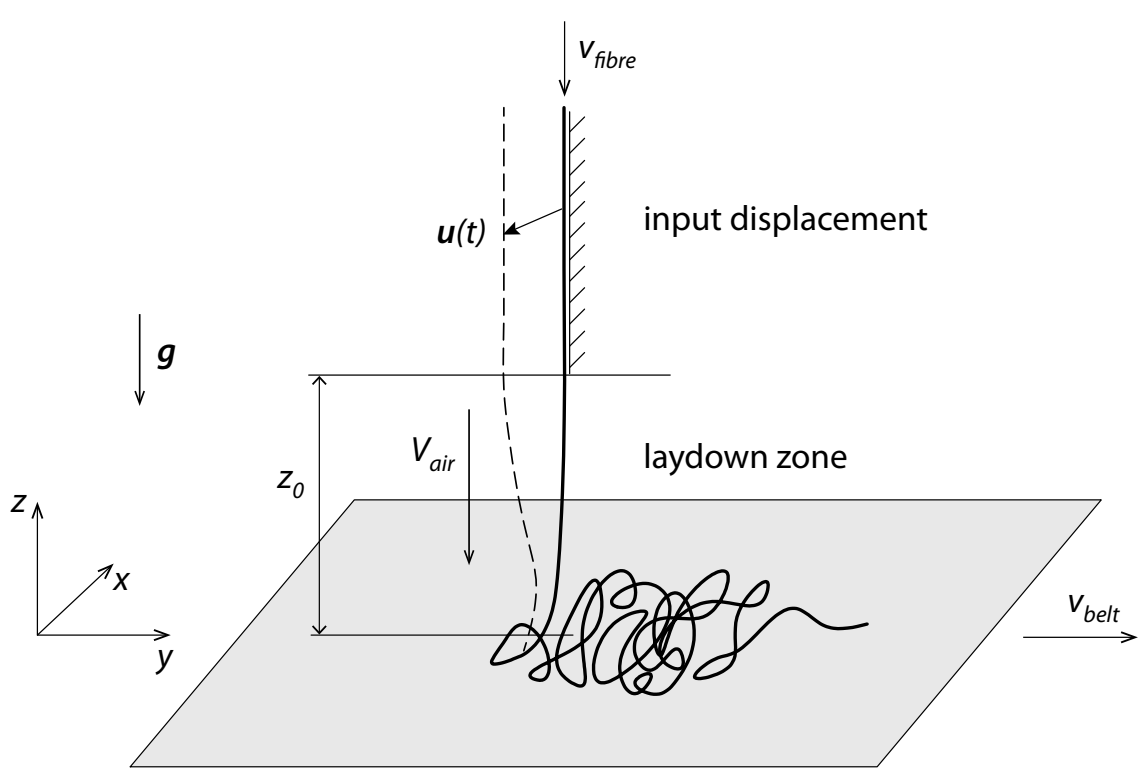

(b)

Figure 1: (a) Schematic of the laydown process in spunbonding, and (b) description of the fibre laydown model.

\section{Single fibre laydown model}

In this section a model is described to simulate the way in which a single fibre lays down onto a moving conveyor belt. The effect of critical model parameters on the form of the resulting fibre geometry is quantified and discussed. 


\subsection{Description of model}

A finite element model of a fibre laying onto a rigid moving surface was developed in Abaqus [27], as illustrated in Figure 1a. A single fibre with length $L=1$ metre was modelled by 1000 three-dimensional first-order Timoshenko beam elements (Abaqus element type B31). Such a fine mesh is necessary to describe accurately the smallest radii of curvature of the loops that the fibre can form during the irregular laydown, and to prevent geometrical stiffness in bending [28]. Material properties used for the fibre were the same as in our previous study with values chosen to represent the industrial process [18]: elastic modulus $E=2 \mathrm{GPa}$, mass density $\rho=950 \mathrm{~kg} / \mathrm{m}^{3}$, Poisson's ratio $\nu=0.3$. The dynamics of the fibre was solved using the non-linear dynamic explicit solver, to account for the fact that a very slender beam may undergo large bending deformations when subject to moderate loads [29]. The explicit solver is also required to cope with the intermittent contact between the fibre and the collecting surface [27]. The description of the model is presented below, including the air drag load formulation, the boundary conditions, and the contact between the fibre and the rigid plane.

Referring to Figure 1, the simulation starts with the fibre aligned vertically, an initial vertical velocity $v_{\text {fibre }}$ at all the nodes, and a gravity acceleration $g=9.81 \mathrm{~m} / \mathrm{s}^{2}$ directed downwards. The top node is constrained to move with constant vertical velocity $v_{f i b r e}$, to respect the fibre continuity within the diffuser, and a laydown zone is defined for $0 \leq z \leq z_{0}$, in which a vertical air flow with velocity $V_{\text {air }}$ produces a drag force on the fibre elements. The formulation of the air drag force on the fibre is the same as used in [18], in which the drag load is decomposed into two components tangential and normal to the fibre orientation. The tangential drag force per unit length is [30] :

$$
f_{t}=\frac{1}{2} \pi d \rho_{a} C_{t} V_{t}^{2}
$$

where $V_{t}$ is the tangential component of the fibre velocity relative to the air flow, $d$ is the fibre diameter, $\rho_{a}$ the density of air, and $C_{t}$ is a skin friction coefficient which is a function of the local Reynolds number $\operatorname{Re}_{t}=V_{t} d / \nu$ given by

$$
C_{t}=\beta \mathrm{Re}_{t}^{-0.61}
$$

where $\beta$ is a constant that depends on the experimental conditions [31]. The normal force per unit length is [32]:

$$
f_{n}=\frac{1}{2} d \rho_{a} C_{n} V_{n}^{2}
$$

where $V_{n}$ is the tangential component of the fibre velocity relative to the air flow, and $C_{n}$ is the normal drag coefficient

$$
C_{n}=A \operatorname{Re}_{n}^{-b}\left(\frac{d}{d_{0}}\right)^{c}
$$

where, for $d=13-390 \mu \mathrm{m}$ and according to [32], the fitted parameters are $A=6.96$, $b=0.440, c=0.404$ and $d_{0}=78 \mu \mathrm{m}$, with the normal Reynolds number defined by

$\operatorname{Re}_{n}=V_{n} d / \nu$, with the viscosity of air $\nu=1.51 \cdot 10^{-5} \mathrm{~m}^{2} / \mathrm{s}$. The air drag force was 


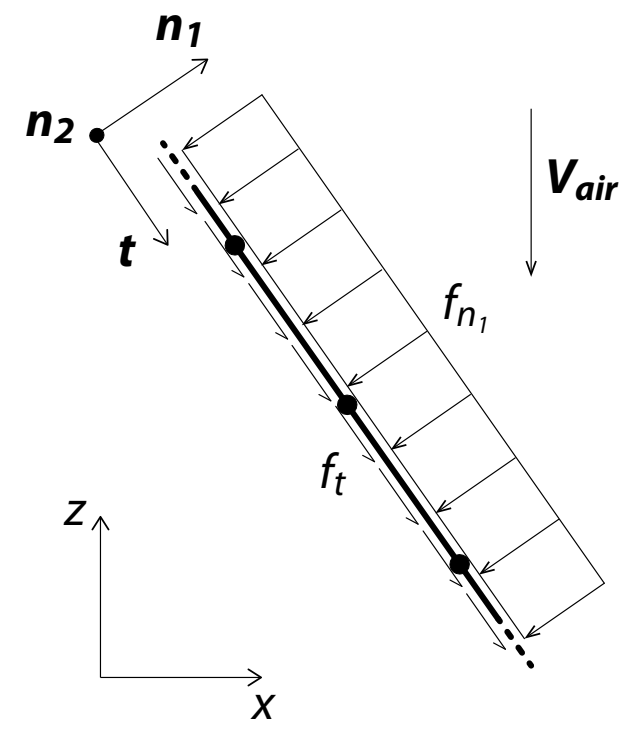

Figure 2: Definition of the air drag load implemented with the user subroutine VDLOAD.

implemented in a $V D L O A D$ Abaqus subroutine, which allows definition of a nonuniformly distributed load along the fibre elements. For each fibre element in the laydown zone, the air-fibre relative velocity is decomposed into the tangential, firstnormal and second-normal directions of the local fibre element frame $\left(\mathbf{t}, \mathbf{n}_{\mathbf{1}}, \mathbf{n}_{\mathbf{2}}\right)$, as shown in Figure 2. The component of the load in the tangential direction was calculated using Equations 1 and 2, while the components in the first and second normal directions were found using Equations 3 and 4.

The fibre-air flow interaction in a spunbonding diffuser produces fibre oscillations whose amplitude and frequency depend on the turbulent flow parameters, as described in detail in $[25,18]$. To simulate this behaviour, an oscillatory transverse displacement $u(t)=u_{0} \sin (\omega t)$ with $\omega=2 \pi f$ and frequency of oscillation $f$, was assigned to fibre nodes above the laydown zone, i.e. with coordinates $z>z_{0}$, as shown in Figure 1 b. The numerical details of how this displacement was imposed are described in the next section. The collecting belt was modelled as a rigid master surface (element R3D4 in Abaqus) moving in the $y$ direction with velocity $v_{\text {belt }}$. A rough contact with Coulomb friction coefficient $\mu$ was included to simulate the tangential forces acting on the fibre from the belt, which arise from the normal air drag force in the laydown zone when the fibre is laying on the belt. It should be noted that in the model the interaction between the air flow and the surface is not included, and the effect of the air suction on the fibre deposition is neglected.

The shape of a fibre laid on to the plane consists typically of an irregular bundle made of coils with different radii of curvature and covering a variable area. In order to represent the results of the simulation, it is helpful to define parameters characterising this shape. As the configuration resembles a long-disordered polymeric chain, this 
suggests characterising the spatial distribution of the fibre by means of the root-meansquare distance of the fibre nodes from the centre of mass of the fibre, which in polymer physics is known as the radius of gyration $S[26]$

$$
S^{2}=\frac{1}{N} \sum_{i=1}^{N}\left(\mathbf{r}_{i}-\mathbf{r}_{O}\right)^{2}
$$

where $\mathbf{r}_{i}$ is the position of the $i$-th fibre node and $\mathbf{r}_{O}$ is the centre of mass of the fibre. The radius of gyration, which defines the coverage area of a fibre on the belt, is not sufficient to describe the smaller scale loops that a slender fibre forms during laydown. If $\theta$ is the orientation of the tangent vector and $s$ the arc-length coordinate along the fibre profile, the curvature $\kappa=\mathrm{d} \theta / \mathrm{d} s$ can be used to describe the fibre geometry at a lower level. The fibre curvature is a crucial parameter in the mechanical behaviour of nonwoven webs, as it can determine the fibre curl between successive bonds, which in turns affects the micro-mechanics of the material. A representative value of the fibre curvature can be defined considering the bending energy stored by the fibre in the deformed configuration:

$$
V_{b}=\int_{0}^{L} \frac{1}{2} E I \kappa^{2} \mathrm{~d} s
$$

which in a non-dimensional form can be expressed as $e=V_{b} L / E I$, where $L$ is the length of the fibre.

\subsection{Details of numerical implementation}

The previous section has given a description of the model, with the relevant governing equations. In this section numerical implementation details are described. Since the fibre starts straight and vertical, the finite element dynamic simulation of fibre laydown needs to model accurately the initial fibre buckling as the fibre move towards the collecting surface. In general, the dynamic analysis of a beam in buckling includes two steps: a static buckling analysis followed by a dynamic post-buckling analysis. Geometric imperfections are generally introduced, based on the results of a modal analysis, to activate the unstable response [27]. However, such a buckling analysis can suffer from numerical instabilities which are computationally expensive to prevent, and which can make the convergence of the solution difficult to achieve.

In reality, for the current laydown problem, initial perturbations to the straight profile arise in the diffuser due to the turbulent air flow, rather than being the result of a buckling instability, as it was previously shown in [25, 18]. This effect was simulated in the laydown model by introducing random imperfections in the fibre profile, namely geometric perturbations of the initial fibre node coordinates, along the $x$ and $y$ directions, with a magnitude of perturbation normally distributed and with a standard deviation of $1 \mu \mathrm{m}$. This solution proved to be effective in seeding the unstable dynamic response of fibre nodes in the laydown zone, i.e. at $0 \leq z \leq z_{0}$, while eliminating the 
numerical instabilities of a buckling analysis, with a consequent dramatic reduction in the computational cost of the simulations. Using different random independent sets of imperfections allowed collection of a statistical set of fibre geometries.

As outlined in the previous section, oscillations in the fibre in the diffuser were modelled by imposing displacements to the fibre nodes above the laydown zone. These boundary conditions were implemented not by imposing the nodal displacements directly, but indirectly via a damped oscillator network as shown in Figure 3a. Dummy nodes associated with the oscillator system were prescribed movement $u(t)$ using the subroutine $V D L O A D$. These dummy node displacements were then transmitted to the fibre nodes through a parallel spring and damper connection. This pragmatic method was found to be the most effective way of assigning nodal displacements which are not bound to the mesh as the fibre moves across the two different zones, one dominated by the fibre oscillation and the other dominated by the air drag. To analyse the indirect implementation of these boundary conditions, consider a one degree of freedom oscillator shown in Figure 3b, in which the displacement $u(t)$ is transferred to the mass through a spring with stiffness $k$ and a damper with constant $c$, which results in a force:

$$
f(t)=-k(x(t)-u(t))-c\left(\frac{\mathrm{d} x}{\mathrm{~d} t}-\frac{\mathrm{d} u}{\mathrm{~d} t}\right)
$$

Assigned oscillatory displacement of dummy nodes $u(t)$ can be effectively rigidly transferred to fibre nodes $x(t)$ for sufficiently high values of $k$ and $c$. For a given nodal mass $m$, optimum $k$ and $c$ values are found by trial and error solving the equation of motion of the one degree of freedom oscillator

$$
\frac{\mathrm{d}^{2} x}{\mathrm{~d} t^{2}}=\frac{1}{m} f(t)
$$

with initial conditions $x(0)=\dot{x}(0)=0$, as in the finite element (FE) fibre model. The best values of $k$ and $c$ were chosen such that the prescribed displacement $u(t)$ corresponded to the displacement $x(t)$ of the 1-DOF oscillator.

Abaqus simulations were run in double precision as a significant improvement of the smoothness of the final fibre profile was observed compared to the single precision analyses. Nevertheless, this increased the computational time and caused numerical instability for a fibre velocity greater than $5 \mathrm{~m} / \mathrm{s}$. Although in the spunbonding process the velocity of the fibre is $30-50 \mathrm{~m} / \mathrm{s}$, the results of simulations undertaken with a smaller velocity of deposition still provide insightful information for nonwovens fibre behaviour. Since the behaviour is dominated by air drag terms [18], the effect of fibre velocity on the general fibre behaviour during laydown is not expected to be great, as discussed in the next section.

Fibre nodal positions at the end of the simulation were saved as a Abaqus results binary file (.fil), and node coordinates were accessed through a dedicated post-processing subroutine $A B Q M A I N$ that converted the binary format into Cartesian coordinates. A mass proportional Rayleigh damping $\alpha_{R}=0.01 \mathrm{~s}^{-1}$ was also included to improve the convergence of the solution [27]. Figure $4 \mathrm{a}$ shows the profile of the fibre after $0.1 \mathrm{~s}$ of simulation for the parameters listed in Table 1 . It can be observed that the fibre profile 


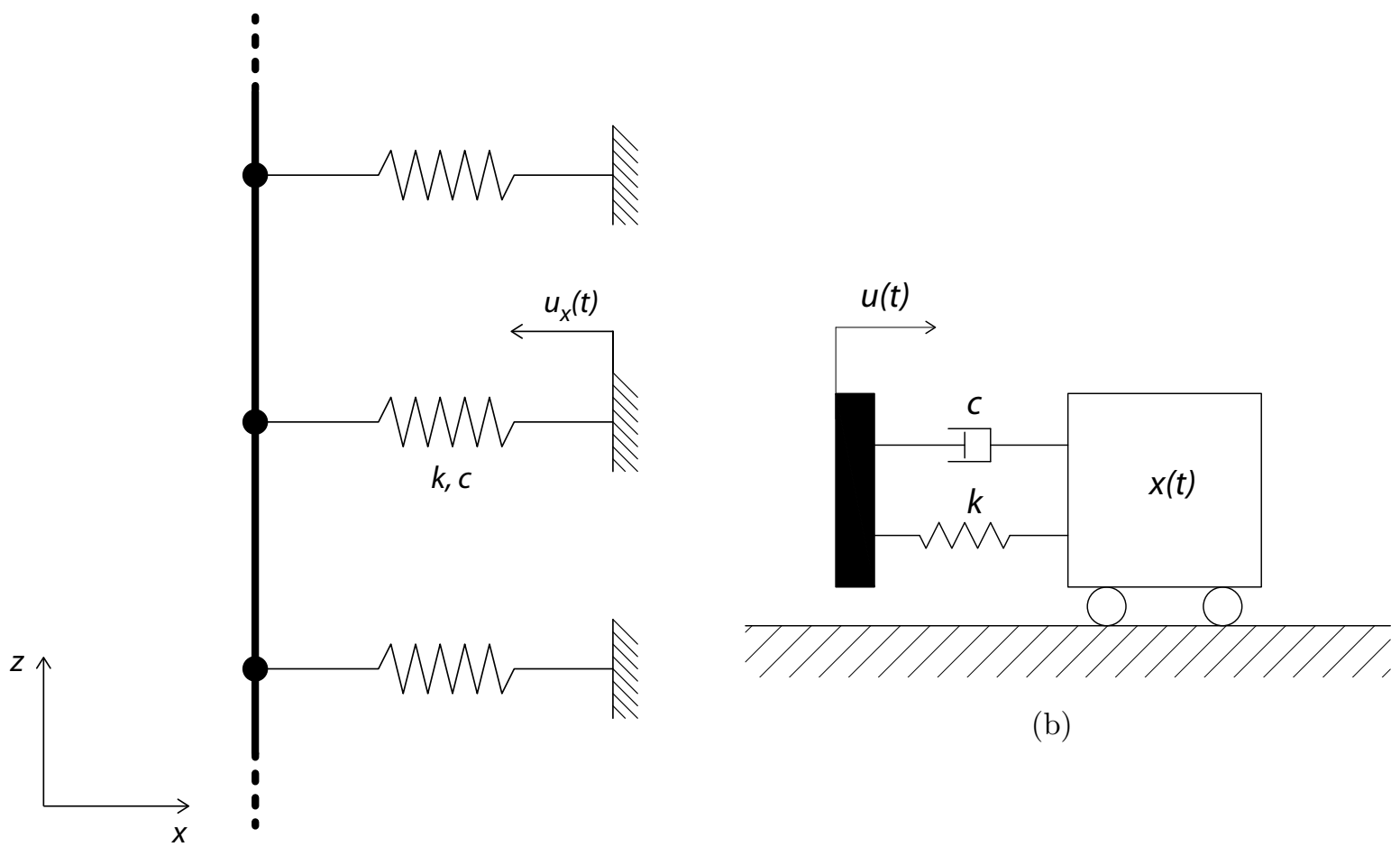

(a)

Figure 3: Definition of forced harmonic displacement; (a) in the fibre finite element model (b) and for a generic single degree of freedom model.

above the laydown zone is perfectly straight, and that the fibre oscillation above the laydown zone matches the imposed input displacement, confirming the effectiveness of the damped oscillator scheme used to impose fibre movement (Figure $4 \mathrm{~b}$ ). This result was obtained using a stiffness $k=1.0 \mathrm{~N} / \mathrm{m}$ and a damping constant $c=0.001 \mathrm{Ns} / \mathrm{m}$ in the forcing harmonic load in Equation 7.

Table 1: Base parameters used for the simulations.

\begin{tabular}{ccccccc}
\hline$v_{f}(\mathrm{~m} / \mathrm{s})$ & $\mu$ & $d(\mu \mathrm{m})$ & $v_{\text {belt }}(\mathrm{m} / \mathrm{s})$ & $V_{\text {air }}(\mathrm{m} / \mathrm{s})$ & $u_{0}(\mathrm{~mm})$ & $\omega(\mathrm{rad} / \mathrm{s})$ \\
\hline 5.0 & 1.0 & 50 & 0.2 & 10 & 10 & 62.8 \\
\hline
\end{tabular}

\subsection{Results of single fibre laydown analysis}

In this section the results of the single fibre laydown model are presented and discussed. All the simulations that follow were undertaken using a fixed feeding height $z_{0}=50$ $\mathrm{mm}$, which is consistent with a typical diffuser-conveyor belt gap in the spunbonding 


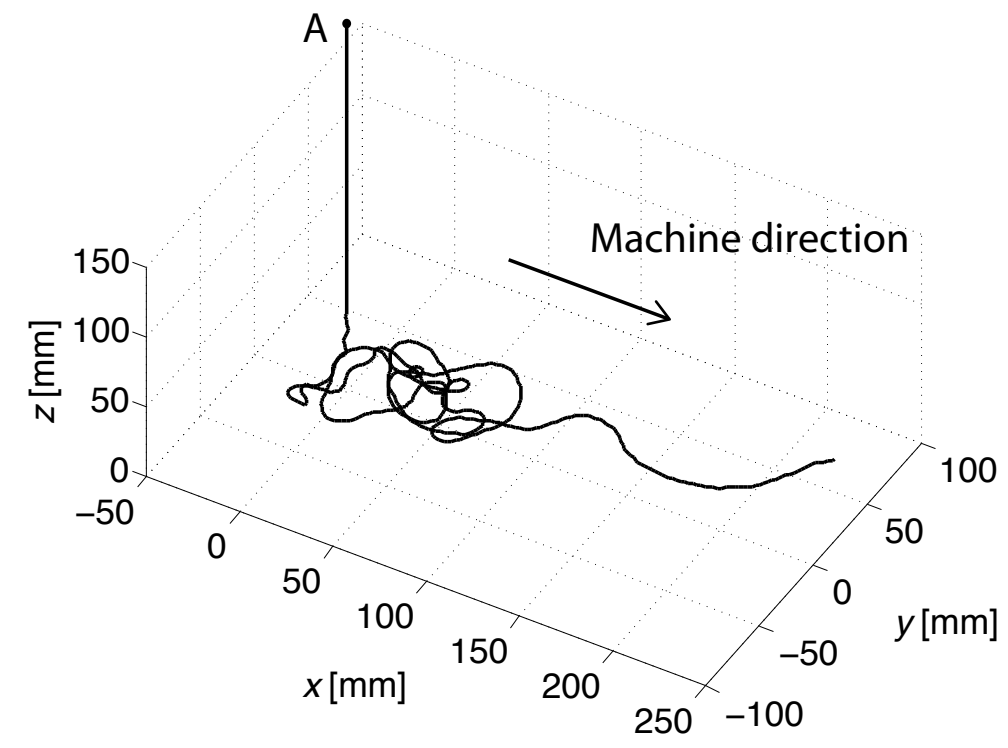

(a)

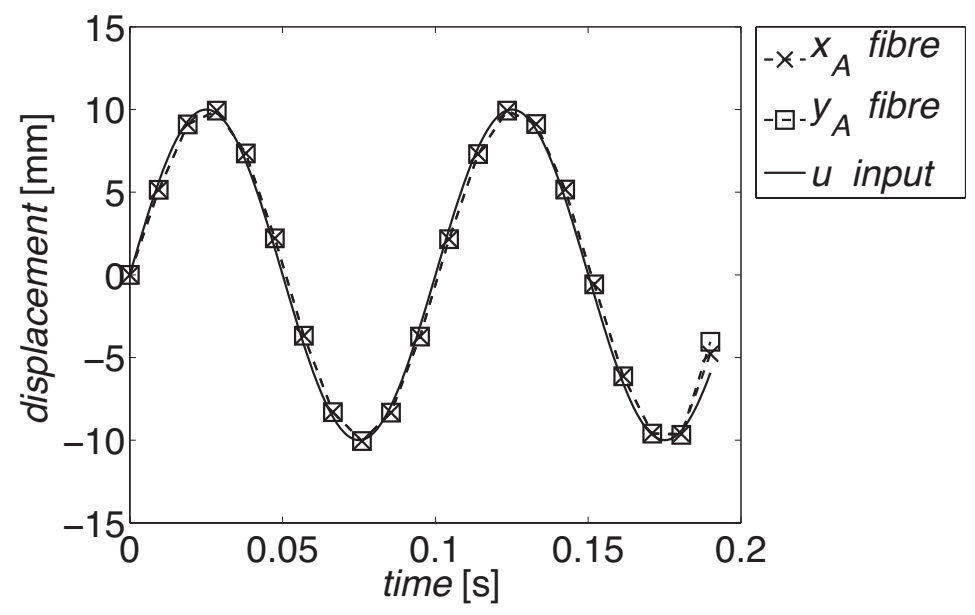

(b)

Figure 4: Fibre laydown numerical simulations; (a) fibre profile after $0.1 \mathrm{~s}$, and (b) comparison between the oscillatory input displacement for $z>z_{0}$ and the resulting fibre node displacement evaluated at node A.

process, and a fibre velocity $v_{f}=5 \mathrm{~m} / \mathrm{s}$. The analyses were terminated when the top fibre node reached the limit of the laydown zone at $z_{0}$, which corresponded to a total period for each simulation of $0.19 \mathrm{~s}$, and a length of deposited fibre of approximately $950 \mathrm{~mm}$. Random perturbations in the initial fibre profile, combined transversal oscillations and vertical air flow, lead to a random-transient dynamic behaviour. To simulate more realistically the industrial process we have deliberately chosen not to include a relaxation step at the end of the analysis. This increased the complexity of the analysis, and as a result in about 10 percent of the simulations we have observed that portions 
of fibre were out of plane as a result of impacts with the normal surface. A simulation was considered valid only when the normal drag force fully prevented bouncing of fibre nodes from the deposition plane, which suggests that the drag term plays a dominant role during fibre deposition.

The effect of friction coefficient, fibre diameter, belt velocity, airflow velocity, and fibre motion, were studied by varying individually the parameters listed in Table 1, with those parameters not varied taking the baseline values given in this Table. For each combination of parameters, ten valid simulations were analysed using a different set of node imperfections to obtain satisfactory statistics of the fibre geometry. The influence of the individual parameters on the fibre radius of gyration $S$ and bending energy $e$ are shown in Figures 5 and 6 . The error bars represent the standard deviation over ten simulations, while the dashed lines indicate values obtained after subtraction of the contribution of the belt motion from the fibre nodes coordinates. The insets in Figure 5 show an example of fibre profile on the belt surface for the smallest value of the parameter investigated, at the top left, and the largest value of the parameter, at the top right.

\section{Effect of friction coefficient}

The effect of the friction coefficient at the fibre-belt contact on the radius of gyration $S$ and bending energy $e$ is shown in Figures $5 \mathrm{a}$ and $6 \mathrm{a}$. Friction forces arise from the normal air drag load which push the fibre towards the horizontal surface. When the contact is smooth the fibre is free to slide over the surface and minimize its bending energy, which leads to large radii of curvature in the fibre profile. By increasing the friction coefficient $\mu$, the instantaneous point of contact is progressively fixed on the plane, and the fibre starts to loop back until, for $\mu>2$, the fibre profile is "frozen" as soon as it touches the surface. As a result, by increasing friction coefficient, $S$ decreases while the bending energy increases, due to the formation of a larger number of small radius loops. In the spunbonding process, fibre sliding is prevented by air suction through a porous belt, see Figure 1a, and the contact forces are significantly different than in this simplified scenario. Nevertheless, it can be assumed that a representative contact formulation in the model is obtained when the fibre stops sliding on the surface. With this in mind, the analysis of the remaining parameters was carried out using as a friction coefficient $\mu=1.0$, which represents the transition between the sliding and sticking regime.

\section{Effect of fibre diameter}

The effect of fibre diameter on the radius of gyration $S$ and bending energy $e$ is given in Figures $5 \mathrm{~b}$ and $6 \mathrm{~b}$. The tendency of a fibre to form loops during laydown is determined mainly by the diameter of the fibre through the bending moment $M_{b}=E I \kappa$, where $I=\pi d^{4} / 64$ is the second moment of area for a circular cross-section. This results in low $S$ and small radius loops for small fibre diameters, and large $S$ and large radius loops at large fibre diameters. It should be noted that the fibre diameter has the largest effect in determining the fibre looping compared to the other simulation parameters. 
Effect of air velocity and belt velocity

The effect of air velocity on the radius of gyration $S$ and bending energy $e$ is given in Figures $5 \mathrm{c}$ and $6 \mathrm{c}$. In the spunbonding process the sliding of fibres during laydown has a negative effect as it leads to formation of non-uniformity in the areal density. As has been shown, fibre sliding can be prevented by a combination of a rough fibre-belt contact and a normal drag given from the vertical air flow passing through the porous belt. However, the plot of $S$ in Figure 5c shows that, since during laydown the fibre is bent, the horizontal component of the air drag load becomes larger, and the fibre is progressively blown away radially, which results in an increase of $S$ with increasing air velocity.

In his pioneering experiments Hearle showed that the motion of the belt modifies the geometry of a thread from a perfect circular coil to a modified cycloid, and eventually to a straight line when the velocity of the thread equals the velocity of the belt. The results in Figures $5 \mathrm{~d}$ and $6 \mathrm{~d}$ confirm that varying the velocity of the belt modifies the geometry of the fibre along the direction of motion of the plane, i.e. the $y$ direction, while the pattern in the cross direction is unmodified, in agreement with Hearle. This is demonstrated by the fact that, after subtraction of the belt displacement, the radius of gyration and the overall fibre curvature are nearly independent of belt velocity.

\section{Effect of fibre oscillations associated with turbulence}

In [18] the authors studied how the turbulent air flow in the spunbonding diffuser can influence the amplitude and frequency of oscillation of a single fibre at the exit of the diffuser. The effect of fibre oscillation before laydown on the resulting fibre laydown geometry is shown for the amplitude of oscillation in Figures 5e and 6e, and for the frequency of oscillation in Figures $5 \mathrm{f}$ and $6 \mathrm{f}$. The amplitude of oscillations $u_{0}$ influences progressively the fibre geometry for $u_{0}>10 \mathrm{~mm}$. In particular, at the highest values of amplitude the fibre pattern no longer consists of irregular coils, but follows the imposed external displacement. The frequency of oscillation, which determines the transverse fibre velocity before laydown, has a marginal effect for a fibre diameter of $50 \mu \mathrm{m}$ due to the resistance of the fibre to bending. However, for a smaller fibre diameter, $d=20 \mu \mathrm{m}$, the fibre is free to follow the imposed displacement, and increasing the frequency of oscillation prevents the formation of small radius loops, as shown by the decrease of $e$ in Figure 6f. 


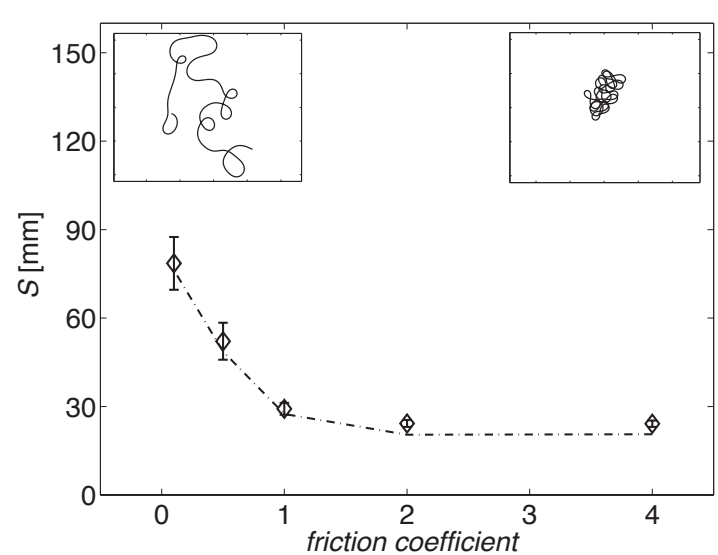

(a)

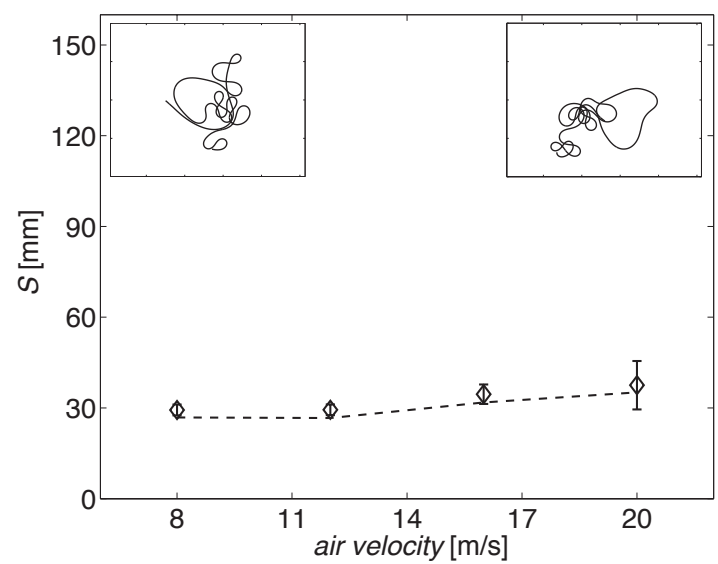

(c)

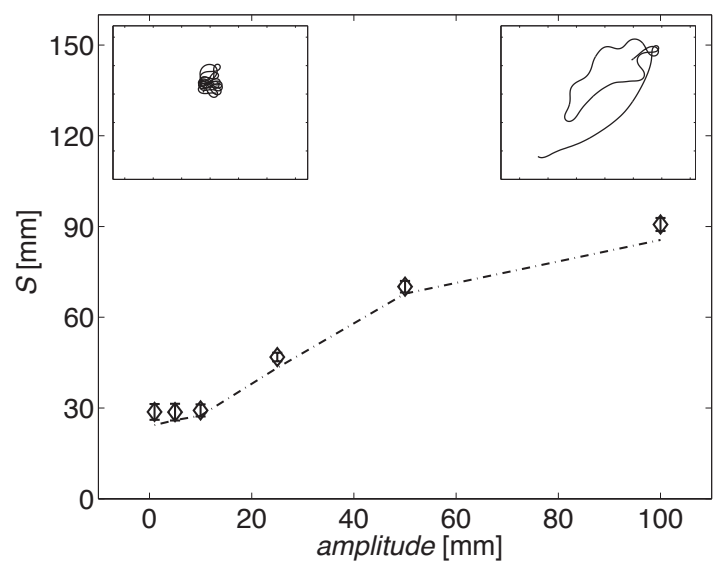

(e)

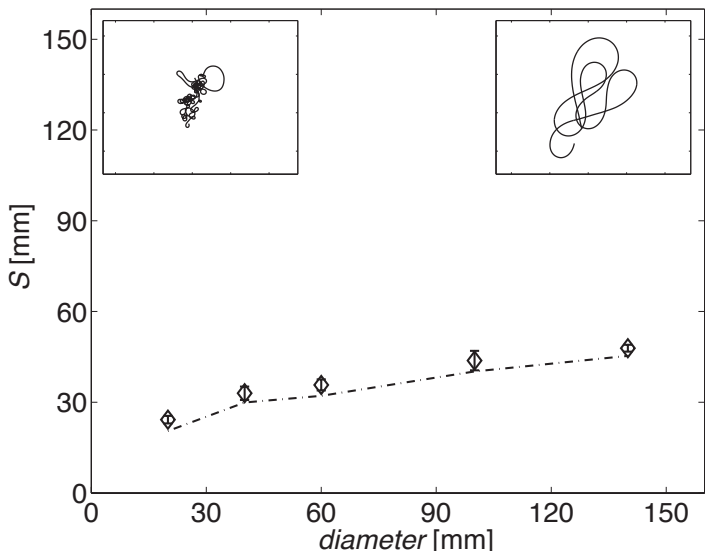

(b)

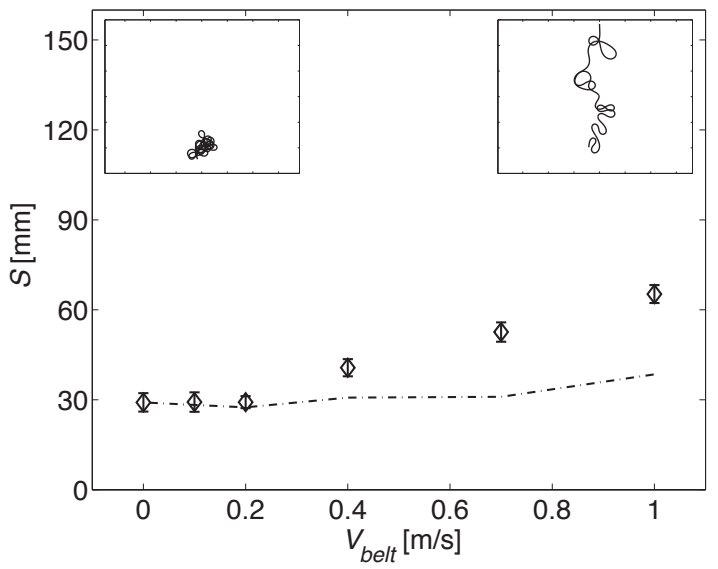

(d)

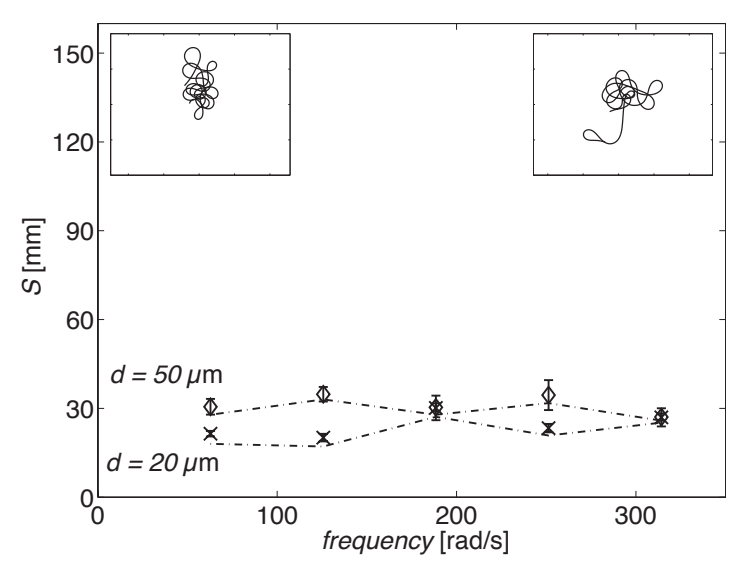

(f)

Figure 5: Radius of gyration $S$ of the laid fibre profile as a function of: (a) friction coefficient, (b) fibre diameter, (c) air flow velocity, (d) conveyor belt velocity, (e-f) amplitude and frequency of fibre oscillation. Insets show the fibre geometry for the lowest and largest values of the relevant parameter (belt direction from bottom to top). Error bars indicate the standard deviation of $S$ over 10 simulations undertaken with different sets of random node imperfections. Dashed lines plot value of $S$ obtained after subtraction of the belt motion contribution. 


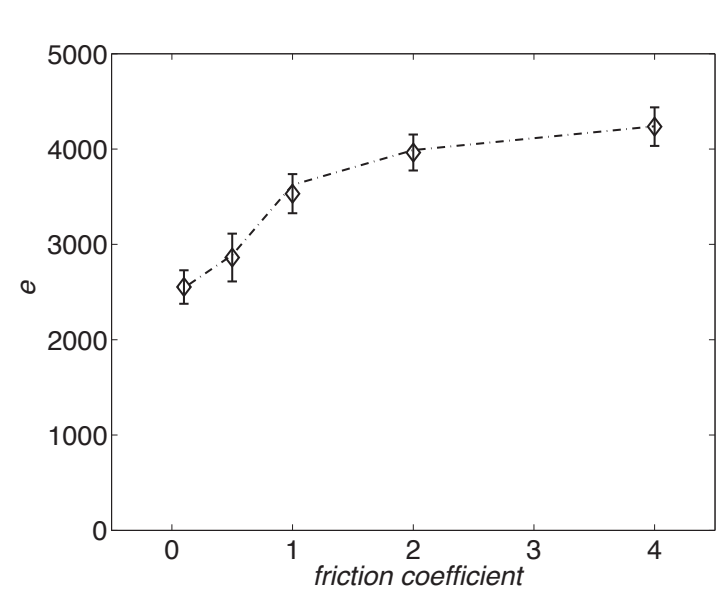

(a)

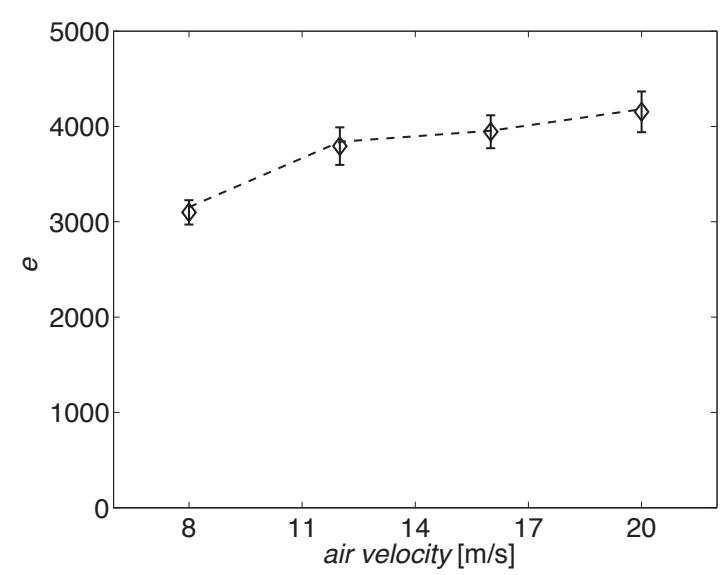

(c)

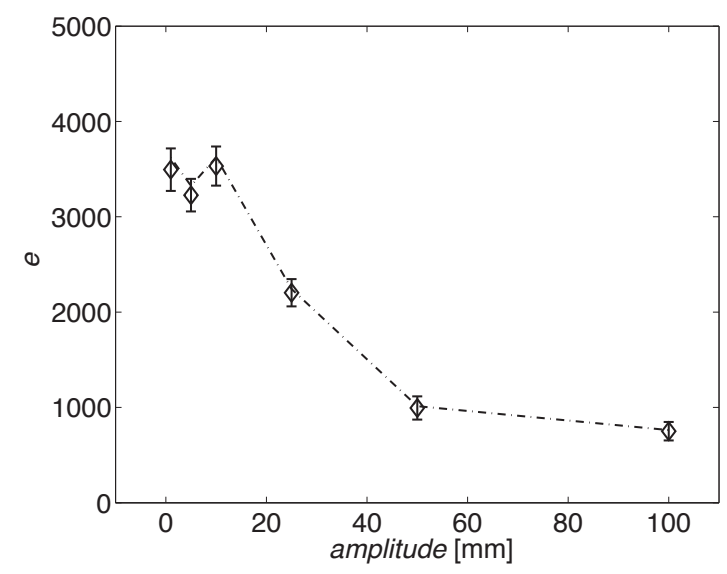

(e)

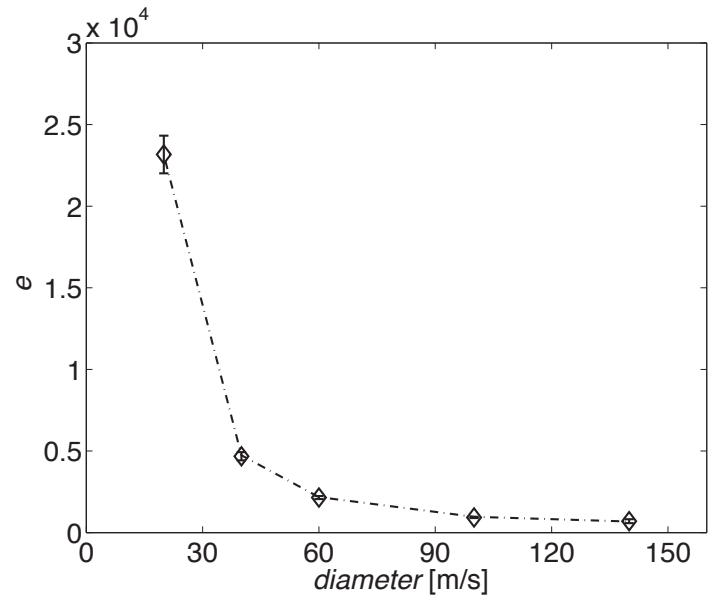

(b)

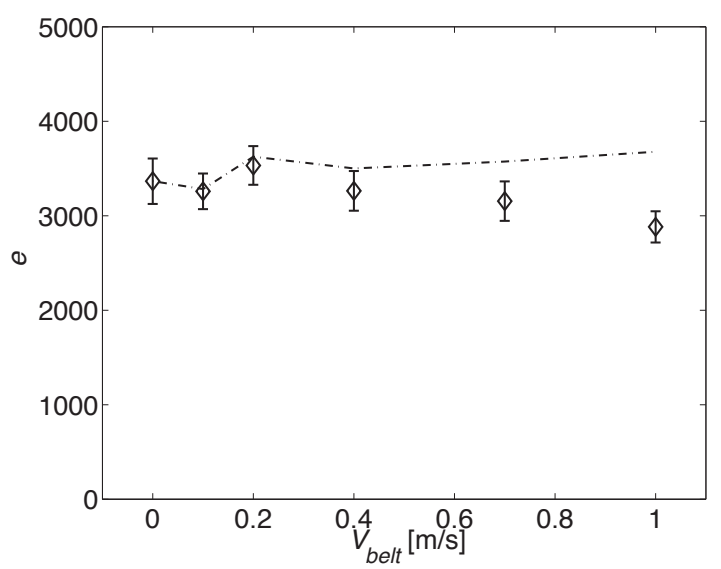

(d)

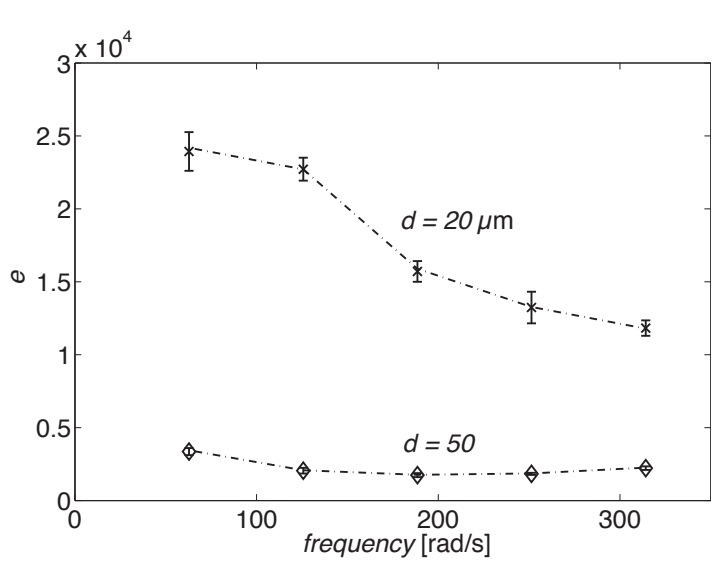

(f)

Figure 6: Non-dimensional bending energy $e$ of the laid fibre profile as a function of: (a) friction coefficient, (b) fibre diameter, (c) air flow velocity, (d) conveyor belt velocity, (e-f) amplitude and frequency of fibre oscillation. Error bars indicate the standard deviation of $e$ across 10 simulations undertaken with different sets of random node imperfections. Dashed lines plot the value of $e$ calculated after subtraction of the belt motion contribution. 


\section{Stochastic modelling of fibre webs}

The results of the previous section have identified the key factors in the dynamics of the single fibre laydown process and the role of these parameters in determining the shape assumed by a fibre on the conveyor belt. A comprehensive study of fibre web formation requires extension of this analysis to a web of many fibres. Simulating the laydown profile of thousands of long fibres through finite element modelling is not feasible as the analysis would be too computationally expensive for practical applications. This section adopts an alternative computationally-inexpensive approach to modelling a full web of fibres. First the finite element model is used to derive a parametric characterisation of the geometry of the fibre profile on the belt. Then a web is generated by superimposing many randomly different realisations of this fibre geometry. The procedure we follow to simulate an artificial web of fibres from stochastically generated fibre curvatures is analogous to the methods used in $[37,33]$ for modelling of stochastic processes.

\subsection{Random fibre generation using a parametric characterisation of geometry}

This section describes a method for generating the geometry of a single fibre from a parametric spectral characterisation of its geometric properties. Here the fibre geometry is modelled as a planar curve lying on the belt. This curve is described mathematically as a function of the arc length $s$ as $\mathbf{r}(s)=(x(s), y(s))$, with local orientation $\boldsymbol{\tau}(s)=\mathrm{d} \mathbf{r} / \mathrm{d} s=(\cos \theta, \sin \theta)$ and curvature $\kappa(s)=1 / \rho(s)=\mathrm{d} \theta / \mathrm{d} s$, where

$\rho$ is the radius of curvature, as shown in Figure 7. By this definition it is possible to reconstruct the geometry of a single fibre $\mathbf{r}(s)$ on the plane if the curvature $\kappa(s)$ is known.

If $s_{m}$ is the discretized arc length of the fibre profile on the conveyor belt

$$
s_{m}=m \Delta s, \quad m=0,1, \ldots, N-1
$$

where $N-1$ is the number of intervals with spacing $\Delta s$, the discrete Fourier transform of the fibre curvature $\kappa_{m}=\kappa\left(s_{m}\right)$ is

$$
K_{r}=\frac{1}{N} \sum_{m=0}^{N-1} \kappa_{m} \exp \left(-2 \pi i \frac{r m}{N}\right), \quad r=0,1, \ldots, N-1
$$

which defines the energy spectrum of curvature $S_{r}=\left|K_{r}\right|^{2}$ in the frequency domain, $\omega_{r}=2 \pi r / L$. By definition $K_{r}$ is periodic with period $N$, and must respect $K_{-r}=K_{r}^{*}$, or $\left|K_{r}\right|=\left|K_{-r}\right|$, where $K^{*}$ denotes the complex conjugate of $K$. For the same energy spectrum, independent random realisations of $\kappa(s)$ can be generated if the phase of $K_{r}$ is modified by a random angle $\theta_{r}$, with uniform probability distribution in the interval $[0,2 \pi]$

$$
K_{r}^{\prime}=\left|K_{r}\right| e^{i \theta_{r}}
$$




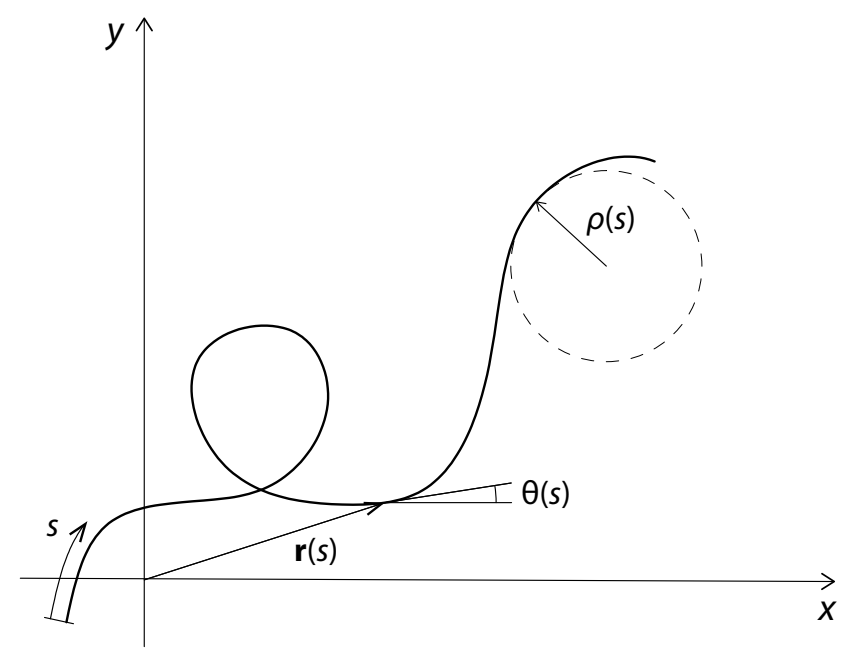

Figure 7: Fibre geometry $\mathbf{r}(s)$ described as a planar curve with arc length $s$, tangent orientation $\theta(s)$ and local radius of curvature $\rho(s)$.

with requirements $\theta_{-r}=-\theta_{r}$ and $\theta_{0}=\theta_{n / 2}=0$ [33]. A randomly simulated fibre curvature is returned by the inverse DFT of $K_{r}^{\prime}$

$$
\kappa_{m}^{\prime}=\sum_{r=0}^{N-1} K_{r}^{\prime} \exp \left(2 \pi i \frac{r m}{N}\right), \quad m=0,1, \ldots, N-1
$$

and a new fibre geometry with the appropriate geometric characteristics can be artificially generated by integrating $\kappa^{\prime}$ twice using the trapezoidal rule:

$$
\begin{aligned}
& \theta_{m+1}=\theta_{m}+\frac{\kappa_{m}+\kappa_{m+1}}{2} \Delta s \\
& \mathbf{r}_{m+1}=\mathbf{r}_{m}+\frac{\boldsymbol{\tau}_{m}+\boldsymbol{\tau}_{m+1}}{2} \Delta s
\end{aligned}
$$

where $\boldsymbol{\tau}_{m}=\left(\cos \theta_{m}, \sin \theta_{m}\right)$. It has been shown in the previous section that, by increasing the belt velocity, the fibre profile is progressively elongated in the direction of the belt motion. If the velocity of the belt is directed along $x$, this effect can be included by defining, similarly to [19]

$$
\frac{\mathrm{d} x}{\mathrm{~d} s}=\cos \theta_{m}+c
$$

where $c$ represents the contribution of the belt motion, which is calculated from the belt velocity $v_{b}$ as

$$
c=\frac{\mathrm{d} x_{b}}{\mathrm{~d} s}=\frac{\mathrm{d} x_{b}}{\mathrm{~d} t} \frac{\mathrm{d} t}{\mathrm{~d} s}=\frac{v_{b}}{v_{f}}
$$

where $v_{f}$ is the velocity of deposition of the fibre on the belt. 


\subsection{Web generation using parametric fibre representation}

This section describes how a random web architecture can be simulated using the parametric representation of the fibre geometry described in the preceding section. Random fibre profiles with the same mesh size and length as for the fibres simulated in the finite element model, were generated from curvature spectra $K_{r}$ of FE simulated fibres by applying Equations 10 to 15. Fibres of arbitrary length were obtained by joining $n_{l}$ randomly simulated fibres, and a web of $n_{f}$ fibres was simulated by assigning as initial positions the coordinates of $n_{f}$ nozzles in a notional spunbonding spinneret regular grid [1]. In this way the fibre geometric parameters derived from the FE analysis are used to build up a series of representative webs without the computational expense of analysing many fibres within the FE environment.

Figure 8 shows the resulting artificially generated webs for fibre diameter $d=20 \mu \mathrm{m}$ and two different amplitudes of oscillation: $u_{0}=1 \mathrm{~mm}$ in Figure 8a, and $u_{0}=50 \mathrm{~mm}$ in Figure 8b. In each figure an example of a single fibre profile is highlighted in bold black. It is seen that at the smaller amplitude of oscillation the fibre forms loops with a small radius of curvature, which is consistent with the results shown in Figure 6e. However, fibre motion along the cross direction (CD) appears to be similar for the two cases, which is not consistent with the results in Figure 5e. This is due to the fact that, by applying Equation 11, the phase of the fibre curvature FFT is lost, which represents a limitation of the current model formulation. Still, Figure 8a suggests a stronger tendency for the fibre to form clusters at small amplitudes of oscillation, which is likely to be a source of web non-uniformity. This phenomenon will be investigated in detail in the next section.

\subsection{Study of web uniformity}

The uniformity of web areal density is a crucial parameter in nonwoven materials, which influences their mechanical behaviour as well as other properties such as filtration, permeability and appearance. It is known empirically that fibre oscillatory motion during deposition is necessary to increase fibre orientation in the cross direction, and to improve isotropy of material properties. However, if the level of turbulence is too high, or if the air suction in the laydown zone is not adequate, fibre motion becomes intensely irregular and web uniformity is compromised. In this section the web simulation methodology described above is used to study theoretically the effect of various process conditions on web uniformity. Predictions are compared with the measured uniformity of real spunbonded nonwoven webs.

The simulated webs are generated using a set of fibres which start out being spaced apart by a regular distance $\Delta w=2.5 \mathrm{~mm}$ along the CD and which then propagate along the MD, as shown in Figure 9. The total length of each fibre is $n_{L} L$, where $L$ is the length of the original fibre, while the width of the starting grid is $2 W=n_{W} \Delta w$. The areal density and the uniformity of a web realisation are evaluated in a square grid with mesh $\Delta h=1 \mathrm{~mm}$ and side $H$. A discrete mass $\Delta m=A \rho \Delta s$ is assigned to each fibre 


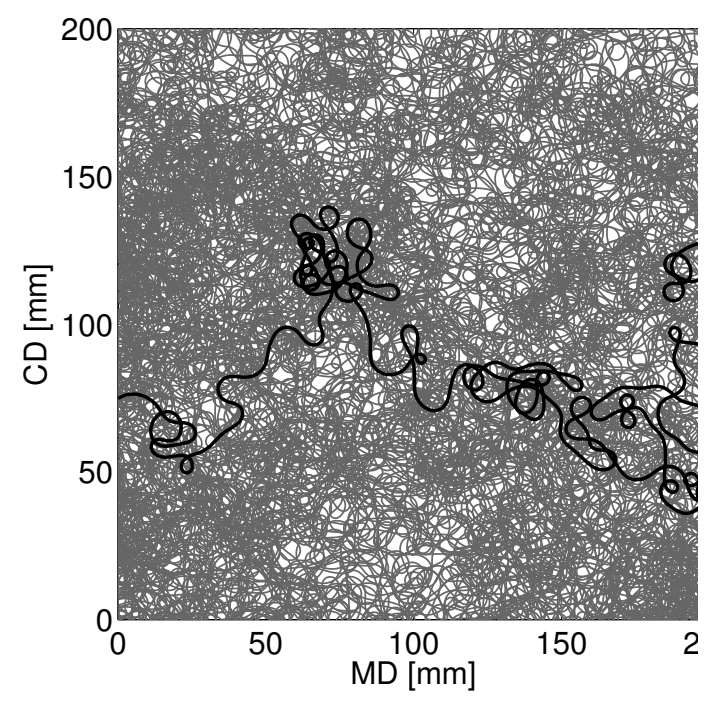

(a)

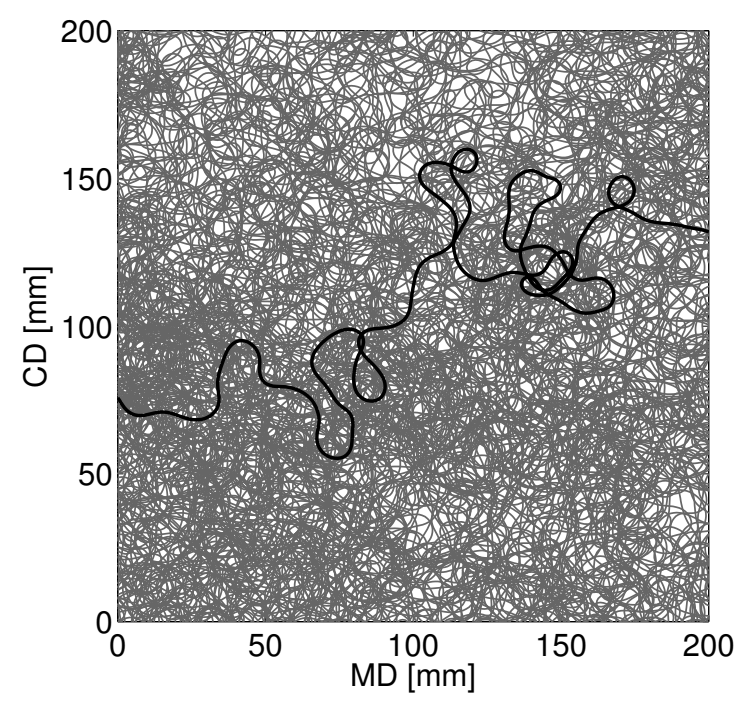

(b)

Figure 8: Stochastic web simulation for fibre diameter $d=20 \mu \mathrm{m}$ and two different amplitudes of oscillation: (a) $u_{0}=1 \mathrm{~mm}$ and (b) $u_{0}=50 \mathrm{~mm}$.

point, with $\Delta s$ the characteristic fibre element size and $A$ the cross-section of the fibre. The total mass of fibre material within the $\mathrm{k}$-th grid element $m_{k}$ is given by summing the individual elements $\Delta m$ within that grid square. The overall web areal density $\rho_{a v}$ is calculated as:

$$
\rho_{a v}=\sum_{k} m_{k} / H^{2}
$$

summing over all the grid elements in the web of side length $H$. A similar expression is used for the local areal density for an individual element of the grid. The uniformity of the web is evaluated in terms of the coefficient of variation $C V$ of the local areal density, defined as the ratio of the standard deviation to the mean of the local areal density, evaluated over all the elements within the mesh grid. The number of fibres, $n_{W}$ and $n_{L}$, in a simulated web must be large enough to cover thoroughly a grid of size $H \times H$ to achieve representative values of web density and uniformity. The corresponding required values of $n_{W}$ and $n_{L}$ were estimated in a convergence study for two different amplitudes of oscillation, $u_{01}=1 \mathrm{~mm}$ and $u_{02}=50 \mathrm{~mm}$, and a grid size $500 \times 500 \mathrm{~mm}$. The results reported in Tables 2 and 3 show that convergence in web areal density and coefficient of variance are reached for $n_{l}>80$ and $n_{w}>1500$. It is also found that the effect of fibre clustering, which takes place predominantly at small amplitude of oscillation (see Figure 8), on web coefficient of variance is minimal, namely $C V \simeq 0.38$ at $u_{0}=1 \mathrm{~mm}$ and $C V \simeq 0.36$ at $u_{0}=50 \mathrm{~mm}$.

To further investigate this aspect, fibre webs were simulated using the idealised square wave spectrum approach described in Appendix A.1 to characterise the fibre geometric 


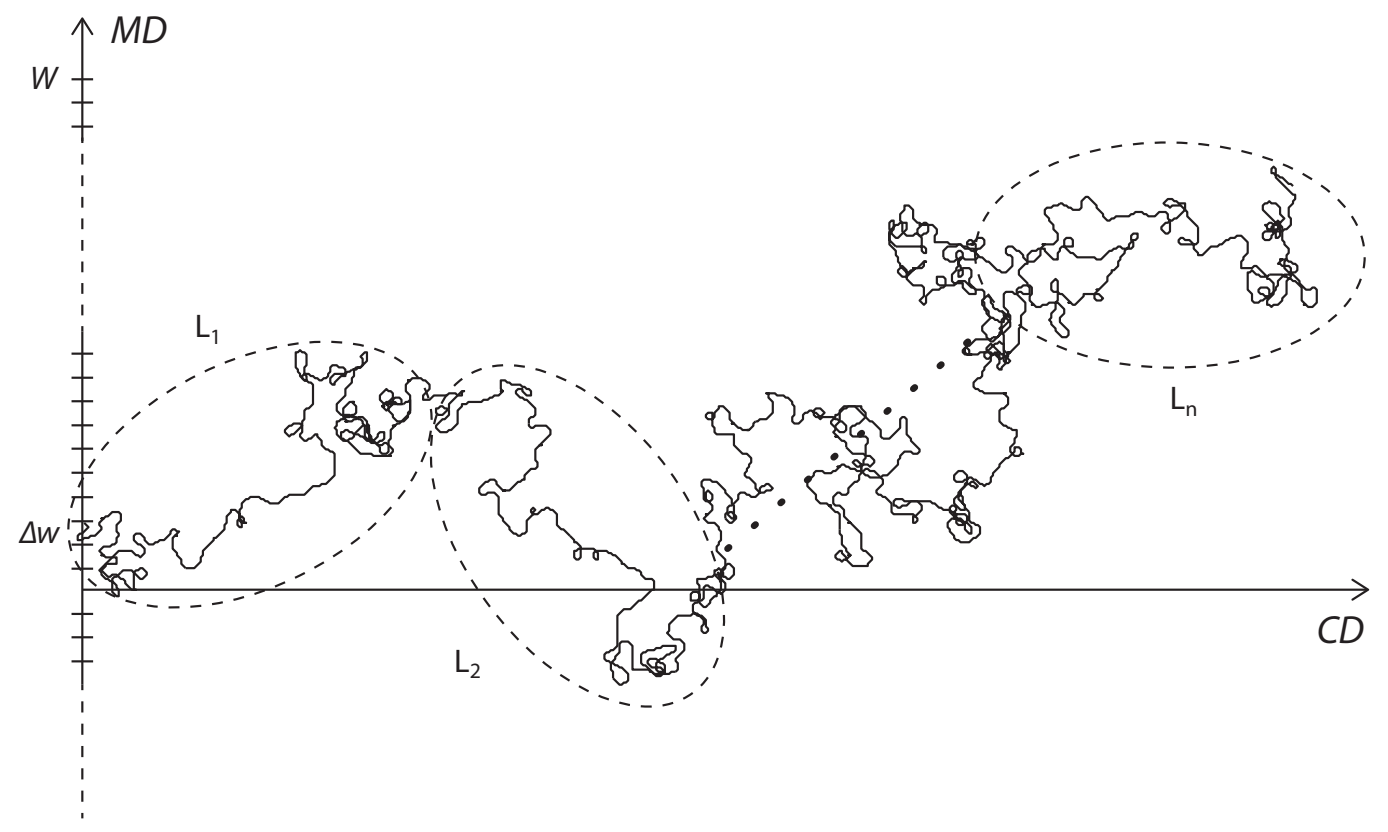

Figure 9: Schematic of the stochastic web generation model.

Table 2: Convergence study at $n_{w}=1500$ : effect of $n_{l}$ on the areal density and uniformity of simulated webs for two different values of amplitude of fibre oscillations and grid size $500 \times 500 \mathrm{~mm}$. Each number represents the average over 10 simulations with standard deviation between parentheses.

\begin{tabular}{llllll}
\hline \multirow{2}{*}{$n_{l}$} & \multicolumn{2}{c}{$\rho_{a v}\left(\mathrm{~g} / \mathrm{m}^{2}\right)$} & & \multicolumn{2}{c}{$C V$} \\
\cline { 2 - 3 } \cline { 5 - 6 } & $u_{0}=1 \mathrm{~mm}$ & $u_{0}=50 \mathrm{~mm}$ & & $u_{0}=1 \mathrm{~mm}$ & $u_{0}=50 \mathrm{~mm}$ \\
\hline 10 & $0.56(0.04)$ & $0.57(0.05)$ & & $1.113(0.039)$ & $0.957(0.049)$ \\
20 & $1.83(0.07)$ & $1.63(0.09)$ & & $0.529(0.018)$ & $0.508(0.023)$ \\
30 & $2.48(0.09)$ & $2.24(0.10)$ & & $0.404(0.010)$ & $0.408(0.009)$ \\
40 & $2.69(0.16)$ & $2.52(0.12)$ & & $0.386(0.011)$ & $0.381(0.012)$ \\
60 & $2.83(0.12)$ & $2.75(0.11)$ & & $0.375(0.010)$ & $0.364(0.009)$ \\
80 & $2.77(0.16)$ & $2.76(0.11)$ & & $0.382(0.013)$ & $0.359(0.006)$ \\
100 & $2.87(0.07)$ & $2.74(0.18)$ & & $0.372(0.007)$ & $0.362(0.011)$ \\
\hline
\end{tabular}

characteristics. Three very different values of bending energy $e$ and cut-off frequency number $n_{c}$ were used, as illustrated by the resulting representative fibre profiles of Figures 10a - 10c. One can observe that, by increasing the bending energy, the radii of loops are decreased, which in turn increases significantly fibre clustering from Spectrum 1 to Spectrum 3. This time the belt-to-fibre velocity ratio $v_{b} / v_{f}$ was also varied via Equation 14.

The effect of belt-to-fibre velocity ratio $v_{b} / v_{f}$ and spectrum on the web overall density 
Table 3: Convergence study at $n_{l}=100$ : effect of $n_{w}$ on the areal density and uniformity of simulated webs for two different values of amplitude of fibre oscillations and grid size $500 \times 500 \mathrm{~mm}$. Each number represents the average over 10 simulations with standard deviation between parentheses.

\begin{tabular}{llllll}
\hline \multirow{2}{*}{$n_{w}$} & \multicolumn{2}{c}{$\rho_{a v}\left(\mathrm{~g} / \mathrm{m}^{2}\right)$} & & \multicolumn{2}{c}{$C V$} \\
\cline { 2 - 3 } \cline { 5 - 6 } & $u_{0}=1 \mathrm{~mm}$ & $u_{0}=50 \mathrm{~mm}$ & & $u_{0}=1 \mathrm{~mm}$ & $u_{0}=50 \mathrm{~mm}$ \\
\hline 250 & $1.60(0.16)$ & $1.20(0.10)$ & & $0.514(0.022)$ & $0.561(0.025)$ \\
500 & $2.38(0.06)$ & $2.11(0.06)$ & & $0.417(0.012)$ & $0.416(0.010)$ \\
1000 & $2.82(0.09)$ & $2.64(0.11)$ & & $0.380(0.009)$ & $0.368(0.009)$ \\
1500 & $2.81(0.08)$ & $2.78(0.12)$ & & $0.377(0.007)$ & $0.361(0.009)$ \\
2000 & $2.84(0.13)$ & $2.84(0.18)$ & & $0.379(0.009)$ & $0.362(0.011)$ \\
2500 & $2.84(0.13)$ & $2.87(0.19)$ & & $0.376(0.016)$ & $0.356(0.012)$ \\
\hline
\end{tabular}

and uniformity is given in Figure $10 \mathrm{~d}$ and 10e. Results show that increasing $v_{b} / v_{f}$ decreases both the density and uniformity for the three fibre spectra. As expected, the web density is independent of the curvature of the single fibres forming the web, whereas the web uniformity decreases from Spectrum 1 to Spectrum 2, and is very similar for Spectra 2 and 3. This result is in agreement with the outcomes of the convergence study above, and suggests that in a web formed of uniformly and independently generated fibres, the uniformity of the web is not determined by the geometry of the individual fibres once a sufficiently large radius of curvature for the loops is exceeded. 


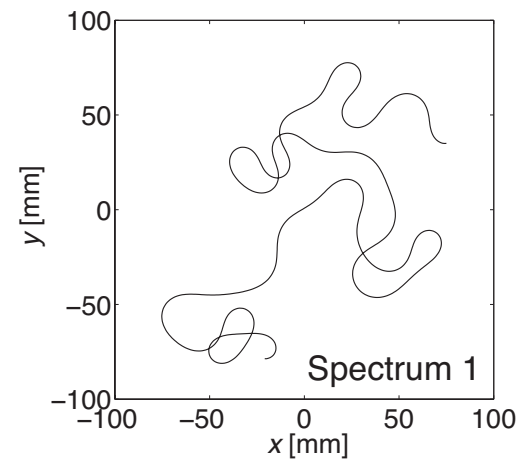

(a)

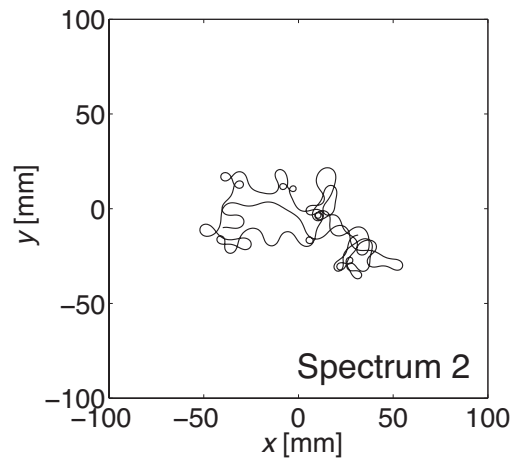

(b)

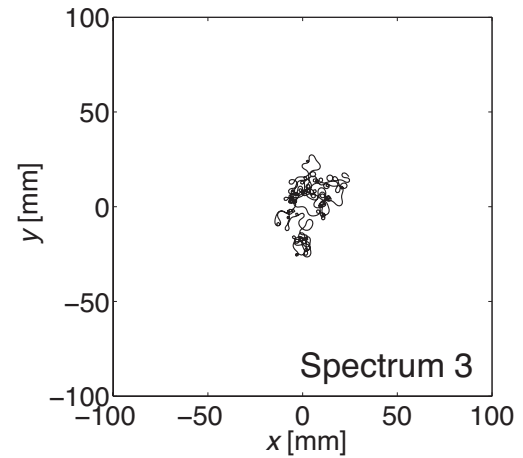

(c)

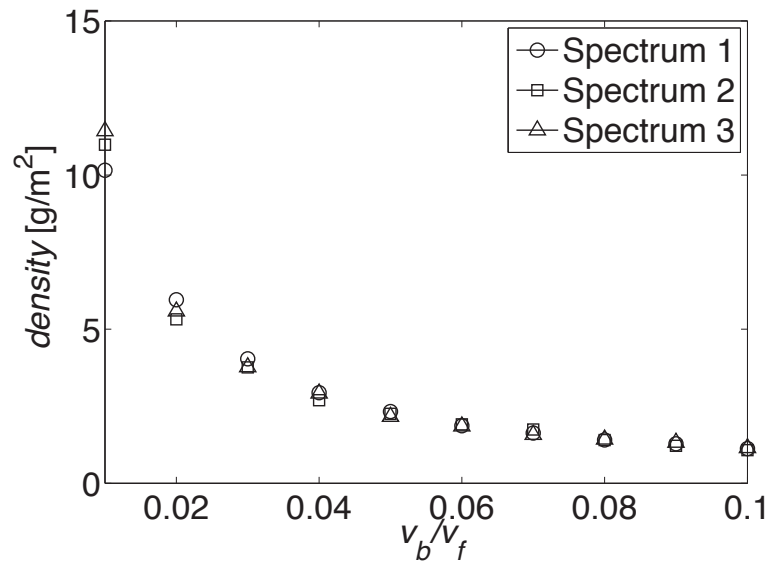

(d)

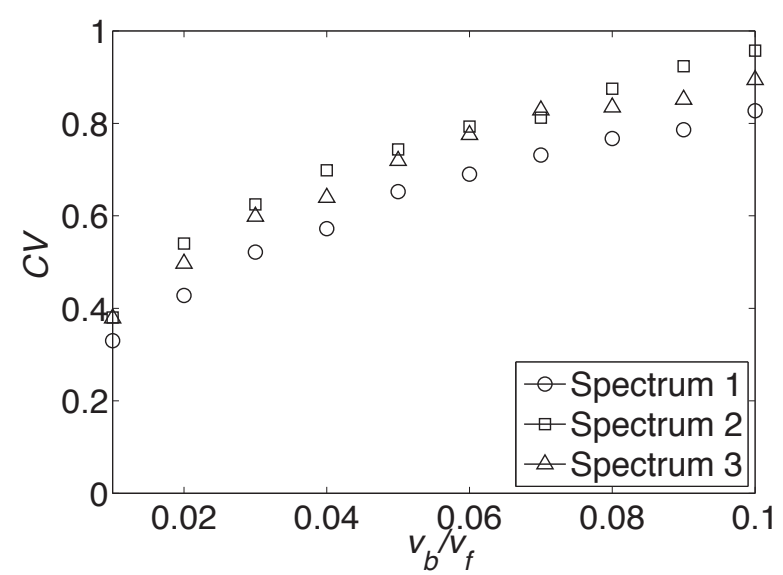

(e)

Figure 10: Example of fibre profiles obtained from square wave spectra; (a) Spectrum1: $e=2.5 \times 10^{3}, n_{c}=20$, (b) Spectrum 2: $e=2.5 \times 10^{4}, n_{c}=50$, and (c) Spectrum 3: $e=2.5 \times 10^{5}, n_{c}=100$. (d) Simulated web density and (e) web uniformity as a function of the belt-to-fibre velocity ratio for the three different spectra. Each data point represents the value averaged over 10 simulations.

\subsection{Measurement of uniformity in real point bonded nonwovens}

This section presents a digital image analysis method which prevents $C V$ biasing due to the influence of the bond pattern in point bonded nonwovens. Grey scale images of polypropylene nonwoven fabrics produced at a spunbonding pilot line using 4 different belt velocities were acquired using an Epson Exp10000XL scanner. Eight web samples of A4 paper size and image resolution $871 \times 617$ pixels were used for each belt velocity. The fibre velocity during laydown $v_{f}$ was estimated using the polymer mass rate $\dot{m}$ and the measured cross-section of the fibre $A_{f}$ through the formula

$$
v_{f}=\frac{\dot{m}}{\rho A_{f} N_{f}}
$$


Table 4: Experimental web data: belt-to-fibre velocity ratio $v_{b} / v_{f}$, fibre diameter, areal density, coefficient of variation of the raw $C V$ and filtered $C V_{\text {corr }}$ images, as an average over 8 measurements, for 4 different thermally bonded nonwovens.

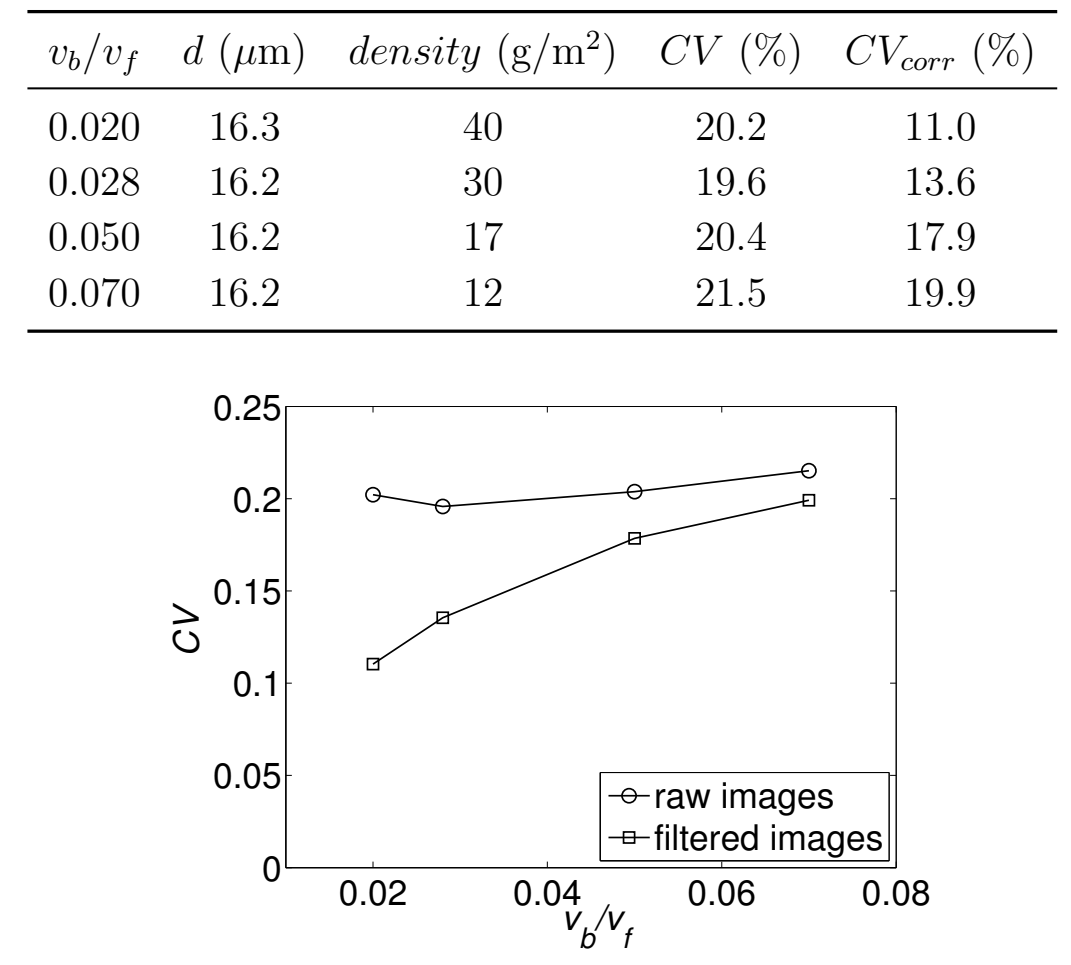

Figure 11: Uniformity of thermally bonded nonwovens as a function of the belt-to-fibre velocity ratio in raw images, and after removing the bond pattern through digital image filtering.

where $N_{f}$ is the total number of fibres, i.e. the number of nozzles in the spinneret. A simple and reliable method to assess the uniformity of nonwovens is to calculate the coefficient of variance of the grey scale level of pixels intensity in a web image $[34,35]$. Although this method is suitable for a loose web, it cannot be applied to point bonded nonwovens due to the regular bond pattern that biases the grey scale uniformity of the image. This issue can be readily noted looking at the data in Table 4 and Figure 11. Decreasing the belt-fibre velocity ratio $v_{b} / v_{f}$ increases the areal density of the web and, as a consequence, a decrease of $C V$ would be expected. However, $C V$ calculated from the grey level of raw images shows a different trend. The influence of the bond pattern is even more severe for high web areal densities, since here the contrast with a dense web can be so intense that the grey level distribution of the image is dominated by the bond pattern, and not by the fibre web.

The problem was solved by eliminating the bond pattern from the raw images before calculating the coefficient of variation of the grey level pixels intensity, through a frequency domain filtering technique. Periodical features of an image are reflected in sharp energy bursts in the two-dimensional energy spectrum of the image [36]. This is 
shown for the raw nonwoven image, Figure 12a, and the corresponding energy spectrum, Figure 12c. The diffuse peak at the centre of the spectrum contains information about the random fibre web, while the sharp peaks are related to the periodic bond pattern. As a consequence, the bonds can be safely eliminated from the original image without losing information on the fibre web if the peaks are deleted from the energy spectrum. This was done routinely in all the web images in a Matlab script that searched and deleted the peaks in the spectrum with a circular notch Gaussian filter, as shown in Figure 12d. A significant improvement for the accuracy of this technique was obtained by fitting and subtracting the broad central peak from the image spectrum before applying the peak-search algorithm. The image of the web without bonds shown in Figure $12 \mathrm{~b}$ is obtained by inverse Fourier transform of the processed spectrum. The spatial features of the images are fully recovered from the image processing technique, although it was observed that, especially in areas with the highest fibre density and at the boundary of the image, the bond pattern might still be partially present after the processing technique. This method can be potentially improved in the future by varying the shape and the dimensions of the notch filter. The coefficient of variation calculated after eliminating the bond pattern, shown in Table 4 and Figure 11, confirms that the uniformity of the fibre web decreases with an increase in the velocity of the conveyor belt.

\subsection{Nonwoven uniformity measurements vs. simulations}

Finally simulations are compared with web uniformity measurement described above. The simulations used a fibre diameter of $20 \mu \mathrm{m}$ and amplitude of oscillation of $10 \mathrm{~mm}$. Such an amplitude of fibre oscillation was observed for a single fibre in a spunbonding diffuser as reported in [25]. The fibre diameter in the real spunbonded webs was in the interval $15-18 \mu \mathrm{m}$, which is slightly lower than in the simulated fibres. However, the results in Figure $6 \mathrm{~b}$ together with the effect of the bending energy $e$ on web uniformity shown in Figure 10, confirms that the analysis is valid. Figures 13a and 13b show the comparison between density and coefficient of variation of simulated and real nonwoven webs as a function of the belt-fibre velocity ratio. It is observed that the density predicted by the model matches the trend of the real nonwoven webs well. The trend of varying web uniformity with velocity ratio agrees well between the measurements and predictions, with the measurements around $13 \%$ higher than in the simulations. This difference could be due to two reasons. The first is associated with the effect of bond pattern on estimating non-uniformity. The image analysis technique described in Section 3.4 attempts to eliminate the bond pattern from the web images but this may not be entirely effective, increasing the measured non-uniformity. The second is that the laydown model assumes that all the fibres are deposited individually, with no interaction between each other. In reality, fibre interaction in the diffuser can take place due to the fibre oscillations, which would result in increased non-uniformity in the fibre laydown. Nevertheless, the agreement is good, both quantitatively and qualitatively. 


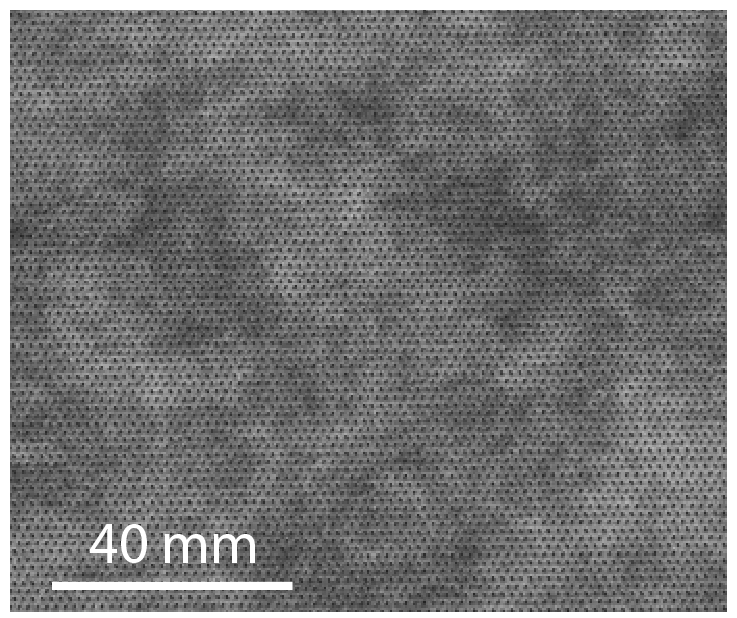

(a)

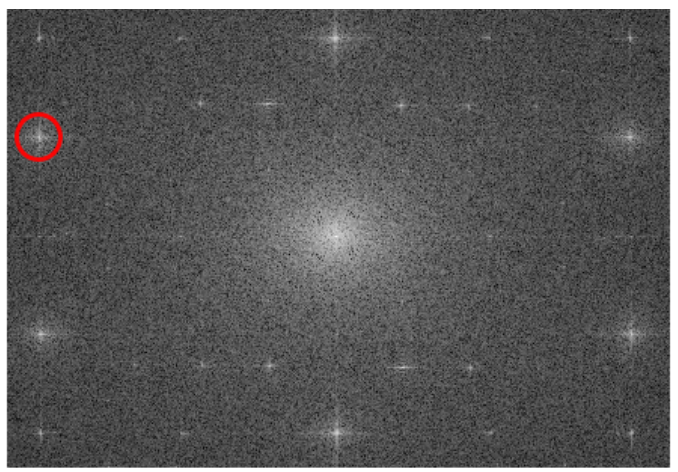

(c)

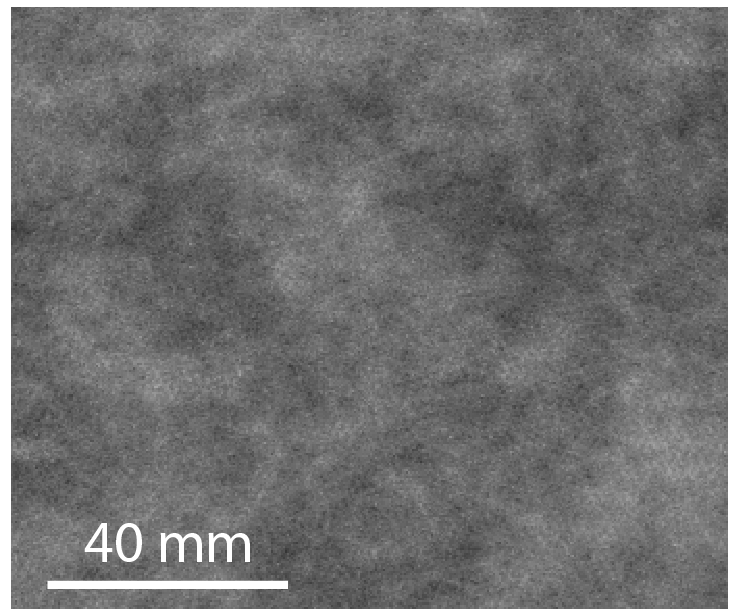

(b)

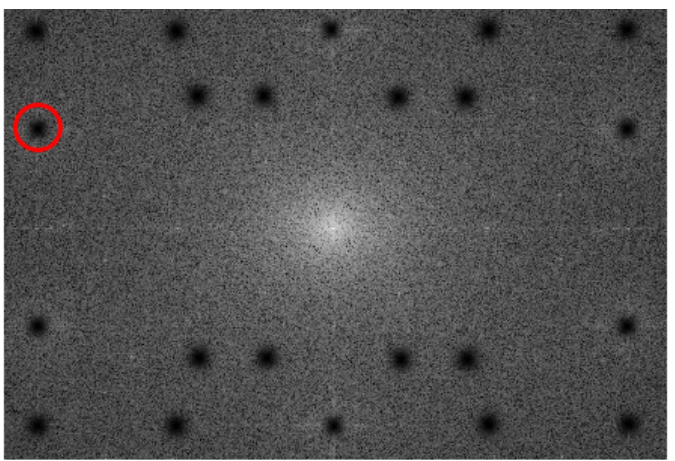

(d)

Figure 12: Web sample obtained with belt-fibre velocity ratio 0.02, (a) raw image, (b) digitally processed image, (c) raw energy spectrum, and (d) energy spectrum with energy peaks associated with the bonds removed.

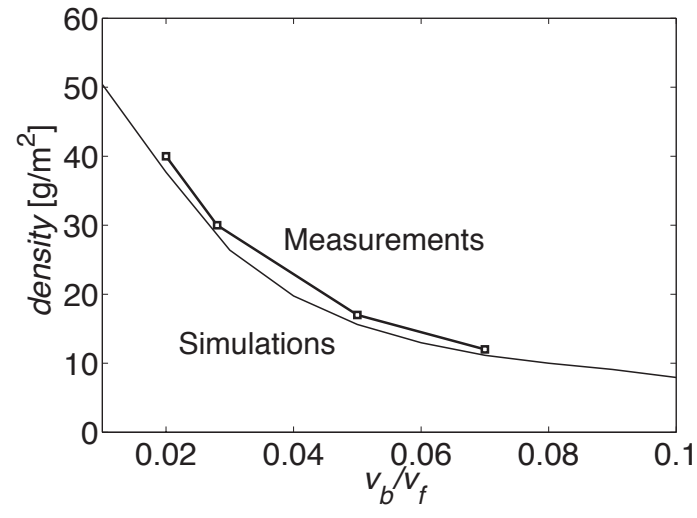

(a)

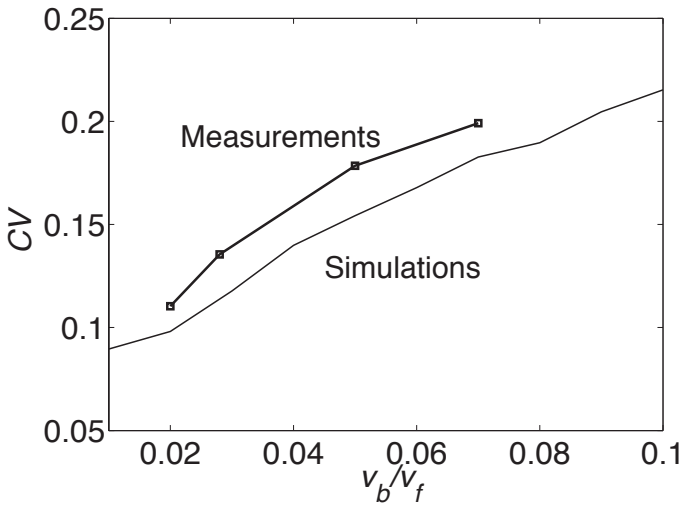

(b)

Figure 13: Comparison between simulated and real spunbonded webs of(a) web density and (b) coefficient of variation. 


\section{Concluding discussion}

The irregular fibre looping path which is drawn by a single fibre during laydown in the spunbonding process can be considered at two different scales. At the large scale the radius of gyration defines the area covered by a single fibre on the web. At the small scale the radius of individual fibre loops governs the tendency of the fibre to form clusters of different areal density and, in point-bonded nonwovens, the free fibre length between successive bonds.

The formation of a regular fibre web requires the tangential forces in the fibre-belt contact to be large enough to prevent slippage of the fibre once it first touches the belt. This condition is achieved through the correct combination of a normal air stream that passes through the porous belt and a rough contact between fibre and belt. If one can assume that fibre slippage is prevented, then the fibre profile is regulated mainly by three parameters: the amplitude of fibre oscillations, which defines the radius of gyration, the fibre diameter, which govern the radius of fibre looping, and the conveyor belt velocity, which elongates the fibre profile along the machine direction.

Despite the fact that a small amplitude of oscillations and a small fibre diameter increase significantly the tendency to fibre clustering, stochastic fibre web simulations have shown that this has a small effect on web uniformity under the fundamental assumption that the fibres are laid down independently from each other. On the other hand, the beltto-fibre velocity ratio is shown to be the main parameter which defines the density and uniformity of the web. This conclusion has been supported by measurements of web uniformity through a digital analysis technique that allows elimination of the bond pattern from web images to avoid biasing of the coefficient of variation. The simulations showed a good agreement with the measurements of web density and $C V$, albeit underestimating the later by about $13 \%$. This relatively minor difference may be due to errors in estimating non-uniformity arising from the bond pattern or due

to fibre interaction in the real process. Further work on the fibre dynamics modelling is required to address the effect of inter-fibre collisions on the web formation. Still, the agreement between measurements and the predictions from the current model formulation is satisfactory and the model provides valuable qualitative and quantitative results to understand the phenomena that govern fibre web formation in the nonwoven fabric production.

\section{Acknowledgements}

The authors are grateful to Fitesa Germany gmbH for financial support and for their help, especially that of Florian Teschner, with providing experimental fabric data. 


\section{Appendix A. Appendix}

\section{Appendix A.1. Random fibre generation from idealised spectral densities}

The random fibre generation method described in Section 3.1 can be generalised if an idealised spectral density function of the fibre curvature $S(\omega)=|K(\omega)|^{2}$ is assumed, instead of using a known fibre profile as a template. For simplicity a square wave function of the form

$$
S(\omega)=\left\{\begin{array}{lll}
S_{0} & \text { if } & |\omega| \leq \omega_{c} \\
0 & \text { if } & |\omega|>\omega_{c}
\end{array}\right.
$$

can be used [33], such that the fibre geometry is fully described by the two parameters $S_{0}$ and $\omega_{c}$. It is known that the energy of a signal in the spatial domain is related to the energy in the frequency domain through Parseval's theorem, which for the fibre curvature reads:

$$
\int_{-\infty}^{+\infty}|\kappa(s)|^{2} d s=\int_{-\infty}^{+\infty}|K(f)|^{2} d f
$$

As a consequence, the non-dimensional bending energy of the fibre

$$
e=\frac{L}{2} \int_{0}^{L} \kappa(s)^{2} d s
$$

is related to the spectral density function through the equality

$$
e=\frac{L}{2} \int_{-\infty}^{+\infty} S(f) d f=\frac{L}{4 \pi} \int_{-\infty}^{+\infty} S(\omega) d \omega
$$

The integral on the right hand side corresponds to the area $2 S_{0} \omega_{c}$ below the square wave function, and gives the relation between the bending energy of a fibre and the parameters that defines $S(\omega)$ :

$$
e=\frac{S_{0} \omega_{c} L}{2 \pi}
$$

where $S_{0}$ and $\omega_{c}$ are respectively the energy density and the cut-off frequency in the square wave spectral density function, and $L$ is the length of the fibre. By increasing $e$ the fibre forms a large number of loops with small radii of curvature, while, for $e$ constant, increasing $\omega_{c}$ leads to a more irregular profile. The latter can be better understood by considering the autocorrelation function of a signal whose spectral density is defined by Equation A.1 [37]:

$$
R_{\kappa}(\varsigma)=2 S_{0} \frac{\sin \omega_{c} \varsigma}{\varsigma}
$$

where here $\varsigma$ represents the lag on the arc length along the fibre. As $\omega_{c}$ increases the curvature becomes progressively uncorrelated for smaller values of $\varsigma$, and the fibre configuration changes from concentric circular loops to uncorrelated irregular loops. This is shown in Figure A1 where four different fibres are generated by varying the bending energy and the cut-off frequency of the energy spectrum. The discrete cut-off frequency is expressed as $\omega_{c}=n_{c} \Delta \omega$ with $\Delta \omega=2 \pi / L$. It can be observed also that, for the same value of bending energy, the fibre geometry is similar to that obtained with the FEM model shown in Figure 5b. 


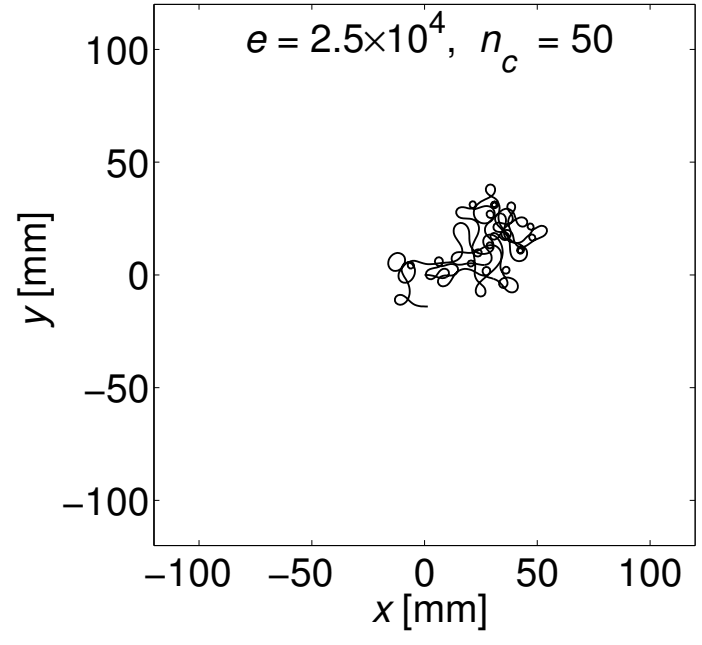

(a)

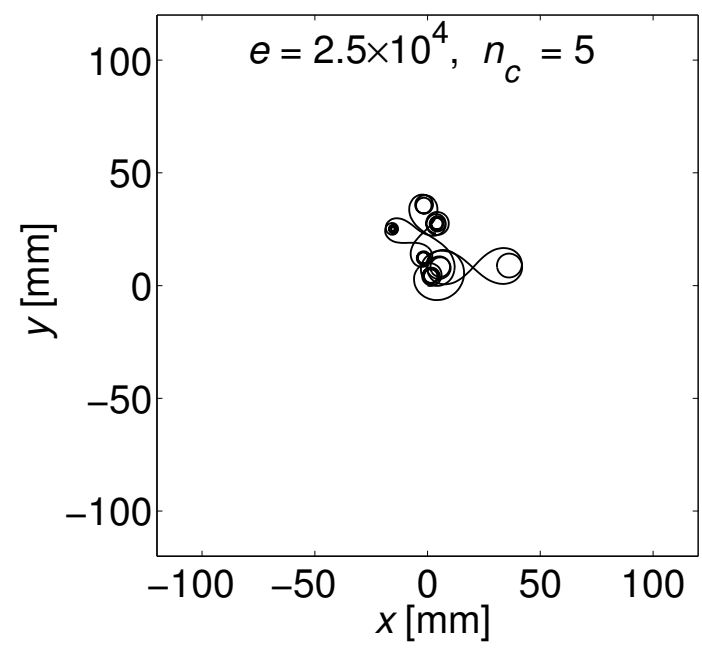

(c)

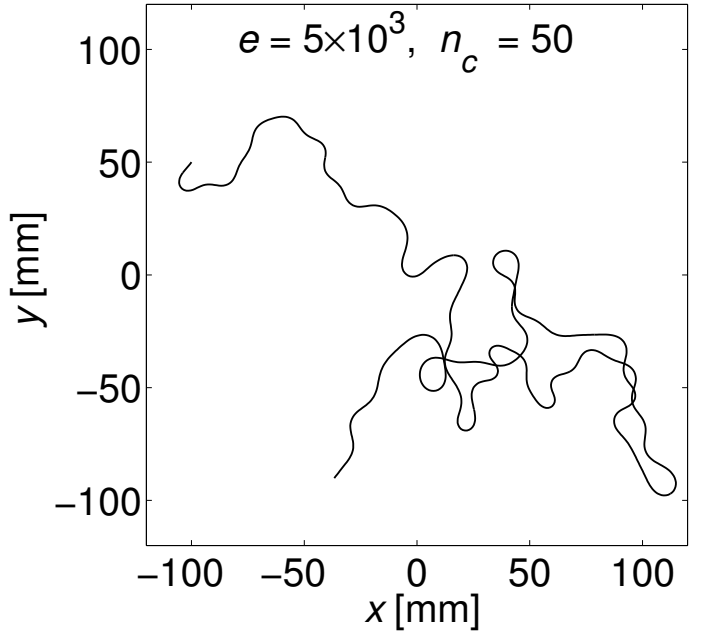

(b)

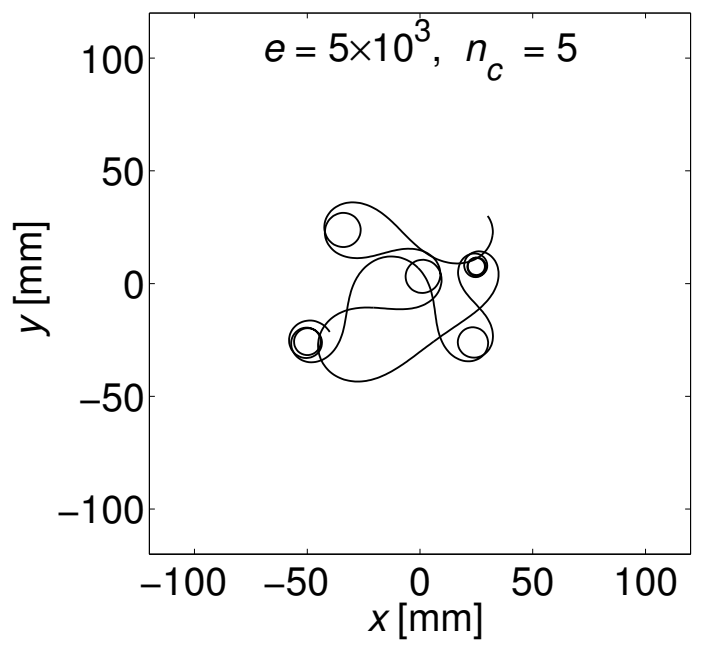

(d)

Figure A1: Fibre profiles randomly generated with a square wave-like spectral density function, Equation A.1, with different values of $e$ and $\omega_{c}$.

[1] Beyreuther R and Brunig H 2010 Dynamics of fibre formation and processing (Berlin: Springer)

[2] Bhat G S and Malkan S R 2002 Extruded continuous filament nonwovens: Advances in scientific aspects J Appl Polym Sci 2002 83(3) 572-585

[3] B Pourdeyhimi, R Ramanathan and R Dent 1996 Measuring fiber orientation in nonwovens. Part I: Simulation Text Res J 66(11) 713-722

[4] B Pourdeyhimi, R Dent and H Davis 1997 Measuring fiber orientation in nonwovens. Part III: Fourier transform. Text Res J 67(2) 143-151

[5] E Demirci, M Acar, B Pourdeyhimi, V V Silberschmidt 2012 Computation of mechanical anisotropy in thermally bonded bicomponent fibre nonwovens Comp Mater Sci 52(1) 157-163

[6] Hearle J W S, Sultan M A I and Govender S 1976 Form taken by threads laid on a moving belt I. Experimental study J Tex I 67(11) 373-376

[7] Hearle J W S, Sultan M A I and Govender S 1976 Form taken by threads laid on a moving belt II. Mechanisms and theory J Tex I 67(11) 377-381 
[8] Hearle J W S, Sultan M A I and Govender S 1976 Form taken by threads laid on a moving belt III. Comparison of materials $J$ Tex I 67(11) 382-386

[9] Mahadevan L and Ryu W S and Samuel A D 1998 Fluid "Rope Trick" Investigated. Nature 392(6672) p. 140

[10] Maleki M and Habibi M and Golestanian R and Ribe N and Bonn D 2004. Liquid Rope Coiling on A Solid Surface Phys. Rev. E 93(21) p. 214502

[11] Chiu-Webster S and Lister J 2006 The Fall of a Viscous Thread Onto a Moving Surface: A FluidMechanical Sewing Machine. J. Fluid Mech 569 89-111

[12] Morris S W and Dawes J H and Ribe N M and Lister J R 2008. Meandering Instability of a Viscous Thread Phys. Rev. E 77(6) p. 066218

[13] Habibi M and Najafi J and Ribe N M 2011 Pattern Formation in a Thread Falling Onto a Moving Belt: an Elastic Sewing Machine. Phys. Rev. E 84(1) p. 016219

[14] Mahadevan L and Keller J B 1996 Coiling of flexible ropes Proc $R$ Soc Lond A 1950(452) 16791694

[15] Antma S S 1995 Nonlinear Problems of Elasticity (Springer Verlag: New York)

[16] Habibi M and Ribe N M and Bonn D 2007 Coiling of elastic ropes. Phys. Rev. E 99(15) p. 016219

[17] Jawed M K and Reis P M 2014. Pattern morphology in the elastic sewing machine Extreme Mechanics Letters 1 76-82

[18] Battocchio F, Sutcliffe M P F and Teschner F 2015 Fibre behaviour in the spunbonding process. Part II: Modelling fibre dynamics in the diffuser. Proc Inst Mech Eng C 231(3) 407-417

[19] Götz T, Klar A, Marheineke N and Wegener R 2007 A stochastic model and associated fokkerplanck equation for the fiber lay-down process in nonwoven production processes SIAM J Appl Math 67(6) 1704-1717

[20] Bonilla L L, Götz T, Klar A, Marheineke N and Wegener R R 2007 Hydrodynamic limit of a fokker-planck equation describing fiber lay-down processes SIAM J Appl Math 68(3) 648-665

[21] Herty M, Klar A, Motsch S. and Olawsky F 2009 A smooth model for fiber lay-down processes and its diffusion approximations Kinetic and Related Models 2(3) 489-502

[22] Marheineke N and Wegener R 2011 Modeling and application of a stochastic drag for fibers in turbulent flows. Int J Mutliphas Flow 37(2) 136-148.

[23] Marheineke N and Wegener R 2006 Fiber dynamics in turbulent flows: General modeling framework. SIAM J Appl Math 66(5) 1703-1726

[24] Oksendal B K 2003 Stochastic Differential Equations: An Iintroduction with Applicatios (Springer: Berlin)

[25] Battocchio F, Sutcliffe M P F and Teschner F 2015 Fibre behaviour in the spunbonding process. Part I: Characterisation of air flow and fibre motion in the diffuser. Proc Inst Mech Eng C

[26] Flory P J 1953Principles of Polymer Chemistry (New York: Cornell University Press)

[27] ABAQUS/CAE 6.9 User's manual (Hibbit, Karlsson \& Sorensen, Inc)

[28] Wang $\mathrm{G}$ and $\mathrm{Yu} \mathrm{W}$. and Zhou $\mathrm{C} 2006$ Optimization of the rod chain model to simulate the motions of a long flexible fiber in simple shear flows. Eur J Mech B-Fluid 25(3) 337-347

[29] Lang H, Linn J and Arnold M 2011 Multi-body dynamics simulation of geometrically exact Cosserat rods Multibody Syst Dyn 25 (3) 285-312

[30] Matsui M 1976 Air drag on a continuous filament in melt spinning T Soc Rheol 20(3) 465-473

[31] Majumdar B and Shambaugh R L 1990 Air drag on filaments in the melt blowing process $J$ Rheol 34(4) 591-601

[32] Ju Y D and Shambaugh R L 1994 Air drag on fine filaments at oblique and normal angles to the air stream Polym Eng Sci 34(12) 958-964

[33] Liu D and Fleck N A and Sutcliffe M P F 2004 Compressive strength of fibre composites with random fibre waviness. J Mech Phys Solids 52(7) 1481-1505

[34] Bresee R R and Daniluk T S 1997 Characterizing nonwoven web structure using image analysis techniques. Tappi Journal 80(7) 133-138

[35] Chhabra R 2003 Nonwoven Uniformity - Measurement Using Image Analysis. International 
Nonwovens Journal 12(1) 43-50

[36] Gonzales R C, Woods R E and Eddins S L 1953 Digital image processing using MATLAB (USA: Gatesmark Publishing)

[37] Newland E D 1993 An Introduction to Random vibrations, spectral and wavelet analysis, 3rd edition (Singapore: Longman House, Longman Scientific and Technical) 